\title{
TROMBOEMBOLISMO PULMONAR: DIAGNÓSTICO E TRATAMENTO
}

\author{
PULMONARY THROMBOEMBOLISM: DIAGNOSIS AND THERAPY
}

Flávia Alvares ${ }^{1}$; Adriana Ignácio de Pádua ${ }^{1}$ \& João Terra Filho²

\begin{abstract}
${ }^{1}$ Médicas Assistentes. ${ }^{2}$ Docente. Divisão de Pneumologia. Departamento de Clínica Médica da Faculdade de Medicina de Ribeirão Preto-USP.

CorResPondÊncia: Flávia Alvares. Divisão de Pneumologia. Departamento de Clínica Médica. Faculdade de Medicina de Ribeirão PretoUSP. Avenida Bandeirantes, 3900. CEP 14048-900. Ribeirão Preto, São Paulo, Brasil. Telefone: (16) 602-2631. Fax: (16) 633-6695. Email: falvares@doctor.com
\end{abstract}

ALVARES F; PÁDUA AI \& TERRA FILHO J. Tromboembolismo pulmonar: diagnóstico e tratamento. Medicina, Ribeirão Preto, 36: 214-240, abr./dez. 2003.

RESUMO - O tromboembolismo pulmonar é uma doença freqüente, porém pouco diagnosticada, fato justificado, em primeiro lugar, por ser doença que apresenta sinais e sintomas pouco específicos, causando demora na suspeita clínica, além disso, apesar de haver vários exames complementares, que podem auxiliar no seu diagnóstico, muitos não estão disponíveis na maioria dos serviços médicos. A demora, no diagnóstico, tem repercussões muito sérias, culminando no aumento da mortalidade de pessoas com tromboembolismo pulmonar. Dessa forma, é fundamental empenhar todo esforço no diagnóstico e no tratamento precoce dessa doença.

Assim, esta revisão tem como objetivos principais estimular a suspeita diagnóstica e orientar a investigação e o tratamento da referida doença.

UNITERMOS - Embolia Pulmonar. Embolia e Trombose. Anticoagulação. Diagnóstico

\section{1- CONCEITO}

Tromboembolismo pulmonar (TEP) consiste na obstrução aguda da circulação arterial pulmonar pela instalação de coágulos sangüíneos, geralmente, oriundos da circulação venosa sistêmica, com redução ou cessação do fluxo sangüíneo pulmonar para a área afetada. Essas condições inter-relacionadas constituem o tromboembolismo venoso (TEV), no qual, a trombose venosa profunda (TVP) é o evento básico e o TEP, a principal complicação aguda ${ }^{(1,2)}$.

\section{2- EPIDEMIOLOGIA}

Os principais dados epidemiológicos sobre TEP, de que dispomos são de literatura estrangeira, princi- palmente norte-americana. Estudos recentes, realizados em comunidades, mostraram uma incidência anual de 60-70 casos/100.000 habitantes ${ }^{(3,4)}$.

A despeito do desenvolvimento científico e tecnológico, ocorrido nas últimas três décadas, o TEP ainda é uma das principais causas diretas de óbito em indivíduos hospitalizados, além de ser a mais freqüente complicação pulmonar aguda nesse grupo de pacientes. Assim, também, a freqüência presumida para diagnóstico clínico de TEP, na década de 90, não foi maior do que há 20 anos atrás ${ }^{(5)}$.

A mortalidade por um evento agudo ocorre, predominantemente, nas primeiras horas de instalação dos sintomas. A mortalidade, em pacientes hospitalizados, varia entre 6 e $15 \%$. O diagnóstico correto e a instituição precoce do tratamento diminuem a mortalidade e 
o risco de recorrência do fenômeno tromboembólico. Para pacientes que se apresentam com instabilidade hemodinâmica ou que apresentem comorbidades associadas, a mortalidade aumenta para 20 a $30 \%(6,7)$.

\section{3- ETIOLOGIA E FISIOPATOLOGIA}

Os fatores de risco do TEV/TEP são aqueles que proporcionam as condições básicas de trombogênese venosa (Tríade de Virchow): estase do fluxo venoso, lesão ou inflamação endotelial e estados de hipercoagulabilidade. Os vários exemplos de condições clínicas, predisponentes ao desenvolvimento de TEV/TEP estão relacionados no Quadro $1^{(8 / 13)}$.

Os trombos estão localizados, principalmente, no sistema venoso profundo $(81 \%)$, sendo que as veias proximais dos membros inferiores (ilíacas e femorais) estão relacionadas com maior risco de TEP. As cavidades cardíacas direitas contribuem com cerca de $19 \%$ dos êmbolos, relacionando-se, principalmente, com as cardiopatias dilatadas e isquêmicas e com as arritmias $(14,15,16)$.

Previamente, o tabagismo era considerado como fator de risco para TEV, o que, atualmente, não tem sido confirmado ${ }^{(13)}$. A idade aumenta exponencialmente $\mathrm{o}$ risco de TEV, mas, ainda, não está clara sua contribuição como fator de risco independente $^{(3,9,12)}$.

Exames para diagnóstico de trombofilia (hereditária ou adquirida) são capazes de detectar alterações (principalmente, a síndrome do anticorpo antifosfolípede e deficiências de antitrombina, fator $\mathrm{V}$ de Leiden, proteína $\mathrm{C}$ ou proteína $\mathrm{S}$ ) em 25 $50 \%$ dos pacientes com TEV ${ }^{(17)}$. Geralmente, essas alterações precisam se associar com fatores de risco adquiridos, para causarem trombose ${ }^{(10)}$. Sendo assim, indica-se realizar os testes para trombofilia apenas nos pacientes com menos de 50 anos que apresentam episódios recorrentes de TEP, sem outro fator de risco evidente, e, em pacientes com história familiar de TEV, com acometimento de mais de

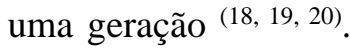

Durante os 12 meses que seguem ao primeiro episódio de TEV, principalmente nos casos recorrentes ou naqueles onde outro fator de risco não foi detectado, há um aumento na incidência de câncer ${ }^{(21,22)}$. Nesses casos, além de uma avaliação clínica (história e exame físico) detalhada, o paciente deverá ser submetido a uma radiografia de tórax e a exames gerais de sangue. Não há indicação para realizar outros exames (ultra-sonografia, tomografia computadorizada,
Quadro 1 - Fatores de risco para tromboembolia venosa (TEV)

Fatores Maiores de Risco (risco relativo 5 a 20)

\begin{tabular}{|l|l|l|l|}
\hline Cirurgia & $\begin{array}{l}\text { Cirurgia abdominal ou pélvica } \\
\text { Prótese de joelho ou quadril } \\
\text { Necessidade pós-operatória de UTI }\end{array}$ \\
\hline Obstétricos & $\begin{array}{l}\text { Gravidez tardia } \\
\text { Parto cesária, pré-eclampsia, multiparidade } \\
\text { Puerpério }\end{array}$ \\
\hline Membros inferiores & $\begin{array}{l}\text { Fratura } \\
\text { Varizes }\end{array}$ \\
\hline Neoplasias malignas & $\begin{array}{l}\text { Abdominal ou pélvica } \\
\text { Avançada ou metastática }\end{array}$ \\
\hline Diminuição da mobilidade & $\begin{array}{l}\text { Hospitalização } \\
\text { Institucionalização }\end{array}$ \\
\hline Outros & Episódio prévio de TEV \\
\hline
\end{tabular}

Fatores Menores de Risco (risco relativo 2 a 4)

Insuficiência cardíaca congestiva Doenças cardíacas congênitas

Cardiovascular Hipertensão arterial sistêmica Trombose venosa superficial Cateter venoso central

Estrógenos Contraceptivo oral Terapia de reposição hormonal

DPOC

Incapacidades de origem neurológica

Tumores ocultos

Doenças trombóticas

Viagens de longa distância

Outros
Doença intestinal inflamatória

Síndrome nefrótica

Diálise

Doenças mieloproliferativas

Hemoglobinúria paroxística noturna

Doença de Behçet 
endoscopia) para rastreamento, a menos que haja alguma evidência clínica que os justifique ${ }^{(23)}$.

Após um evento de TEP, ocorrem alterações respiratórias e hemodinâmicas, estando a mortalidade freqüentemente relacionada às complicações cardíacas e não necessariamente às pulmonares.

\section{1- Conseqüências respiratórias}

Imediatas: aumento do espaço morto do compartimento alveolar, efeito shunt, broncoconstrição e pneumoconstrição associadas, respectivamente, ao aumento da resistência de vias aéreas e redução da complacência pulmonar, distúrbio difusional, hipoxemia, hipo ou hipercapnia, taquipnéia (Figura 1).
Tardias (após 18 h): redução da produção do surfactante, com tendência ao colapso alveolar e edema pulmonar, aumento da resistência de vias aéreas e diminuição da complacência pulmonar, distúrbio difusional, distúrbios $\mathrm{V}_{\mathrm{A}} / \mathrm{Q}$, hipoxemia, taquipnéia (Figura 2).

\section{2- Conseqüências hemodinâmicas}

Redução do leito arterial pulmonar, aumento da resistência vascular, aumento na pressão da artéria pulmonar, aumento da pós-carga e do trabalho do ventrículo direito (ocasionando até sua falência e Cor pulmonale), queda do volume/minuto circulatório, taquicardia, diminuição da perfusão coronariana, isque-

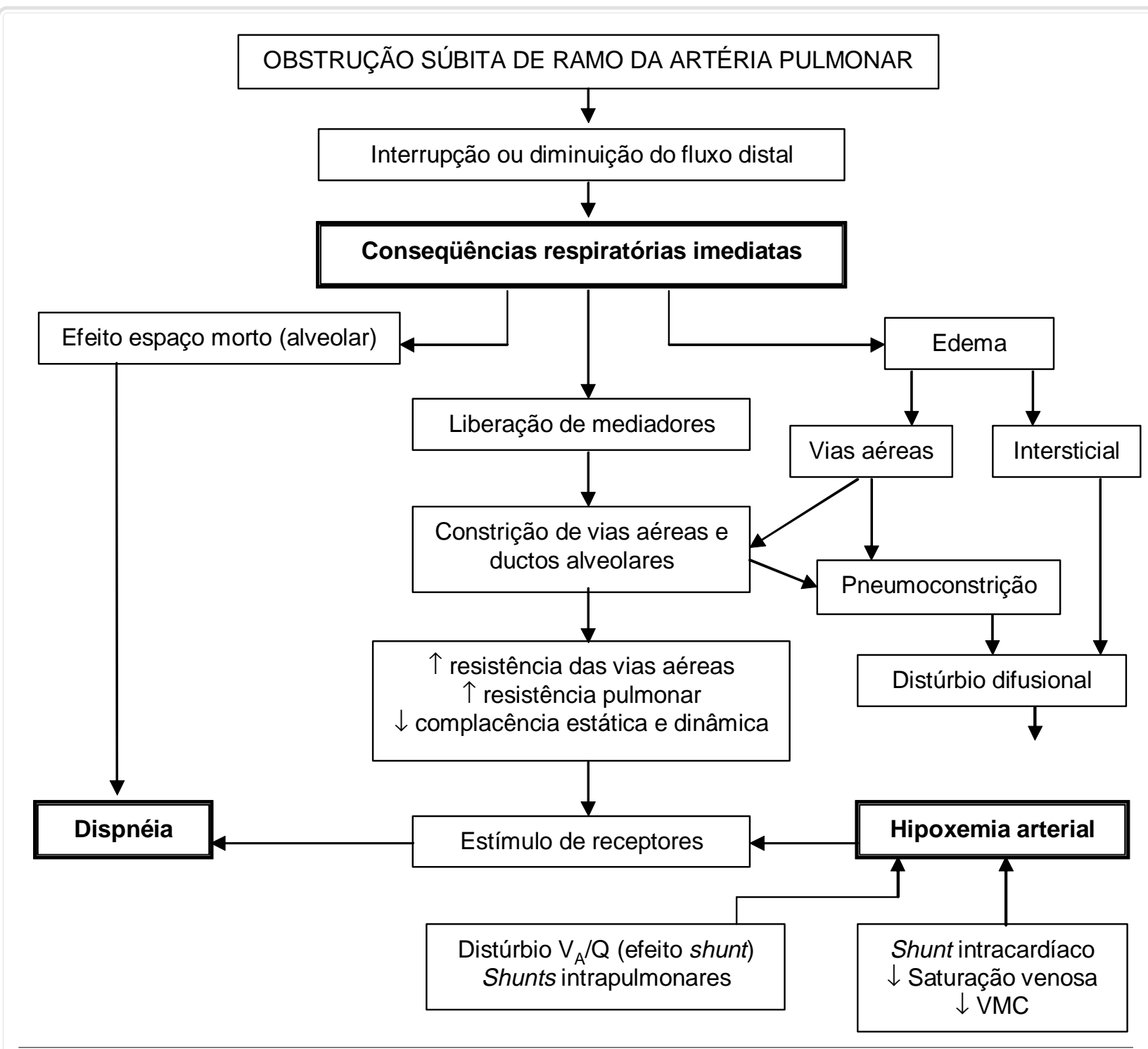

Figura 1 - Fisiopatologia do TEP - Conseqüências respiratórias imediatas 


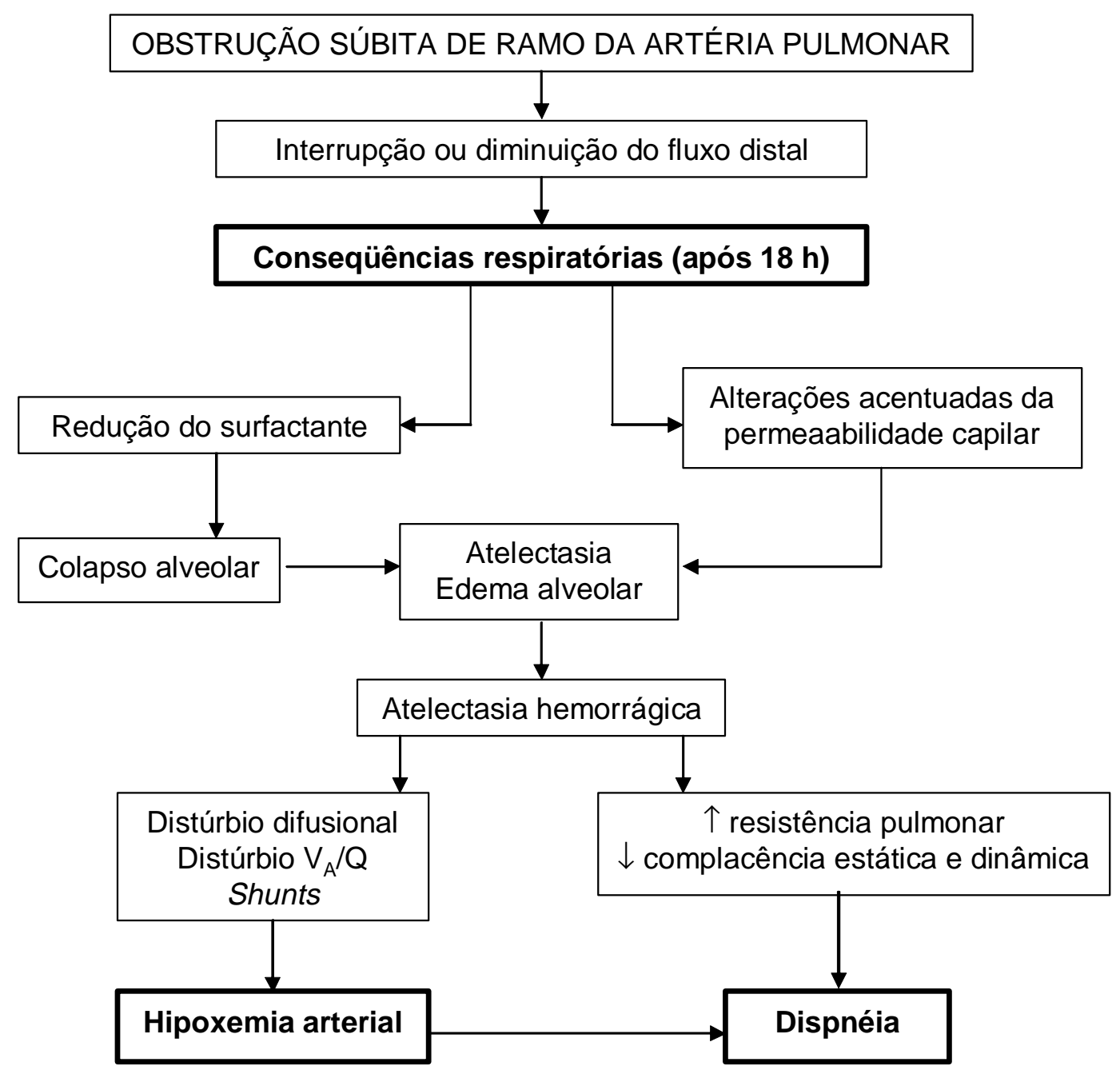

Figura 2 - Fisiopatologia do TEP - Conseqüências respiratórias (após 18 horas)

mia miocárdica, prejuízo de enchimento do ventrículo esquerdo com sua posterior disfunção e até choque circulatório (Figura 3).

Assim, o quadro clínico e sua gravidade irão depender da carga embólica, das condições cardiopulmonares prévias e da capacidade de resposta humoral.

\section{4- MANIFESTAÇÕES CLÍNICAS}

O TEP pode apresentar-se, clinicamente, de várias maneiras e, com grande frequiência, de forma silenciosa.

Os sinais e sintomas presentes em casos comprovados de TEP, quando analisados isoladamente, variam em frequiência, nas diversas séries, trazendo grande dificuldade diagnóstica, sobretudo por serem inespecíficos e por mimetizarem outras condições mórbidas. Os principais sinais e sintomas estão listados no Quadro $2^{(24,25,26)}$.

O TEP, geralmente, manifesta-se sob a forma de um dos seguintes quadros sindrômicos: dispnéia aguda, infarto pulmonar, cor pulmonale agudo, choque cardiogênico e morte súbita.

$\mathrm{Na}$ forma de dispnéia aguda, o quadro é muito inespecífico e exige grande suspeição clínica para o diagnóstico. Outros sintomas e sinais podem se associar em maior ou menor freqüência. A presença de fatores de risco para TEP deve ser considerada para reforçar a possibilidade diagnóstica. 


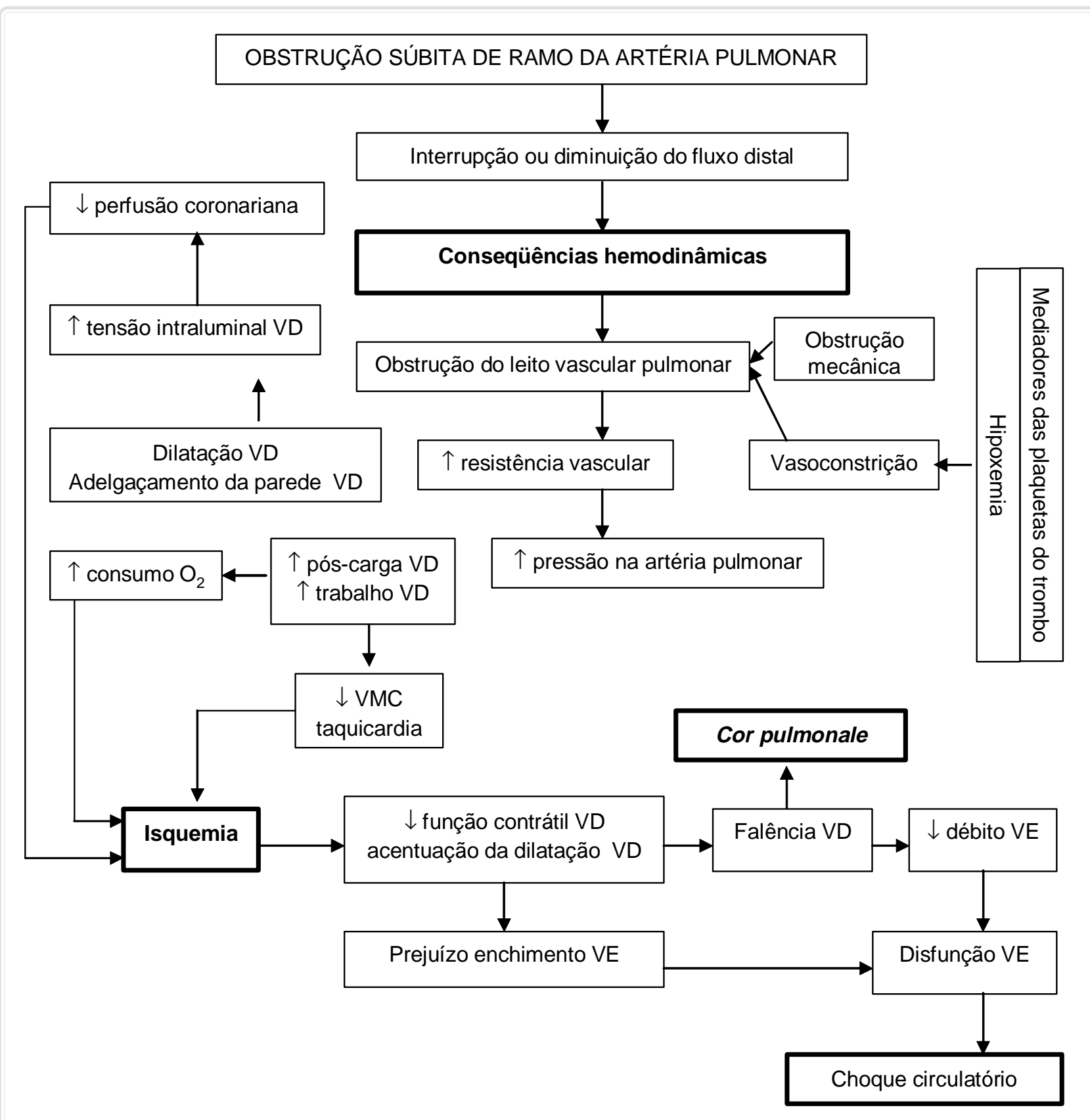

Figura 3 - Fisiopatologia do TEP - Conseqüências hemodinâmicas

No infarto pulmonar, o indivíduo, geralmente, apresenta dispnéia, taquipnéia, dor torácica pleurítica e tosse com hemoptise. O exame físico do tórax pode revelar discretos estertores, sibilos ou sinais de pequeno derrame pleural.

$\mathrm{O}$ cor pulmonale agudo pode manifestar-se de forma isolada ou associado ao choque cardiogênico. Quando isolado, podemos observar sinais de hipertensão pulmonar e dilatação de câmaras cardíacas direitas, associadas ou não às manifestações clínicas de insuficiência cardíaca com congestão venosa. Sua forma mais grave está associada ao choque cardiogênico. Ocorre, quando há obstrução de 50-70\% da circulação pulmonar ${ }^{(27,28)}$, ou mesmo em níveis menores, em pacientes com doença cardiopulmonar prévia ${ }^{(29)}$. O paciente apresenta-se com taquicardia, hipotensão, palidez, sudorese fria, turgência jugular, terceira bulha, hiperfonese de P2. Pode haver síncope ou mesmo morte súbita. 


\begin{tabular}{l|c|c|}
$\begin{array}{l}\text { Quadro 2 - Sinais e sintomas em pacientes com TEP confirmado } \\
\text { por arteriografia. }\end{array}$ \\
\hline Sinais e Sintomas & UPET/USPET (\%) & PIOPED (\%) \\
\hline Dispnéia & 84 & 73 \\
\hline Dor pleurítica & 74 & 66 \\
\hline Tosse & 50 & 37 \\
\hline Dor nos membros inferiores & 39 & 26 \\
\hline Hemoptise & 28 & 13 \\
\hline Freqüência respiratória $>20$ ipm & 85 & 70 \\
\hline Estertores crepitantes & 56 & 51 \\
\hline Freqüência cardíaca > $100 \mathrm{bpm}$ & 58 & 30 \\
\hline Hiperfonese de P2 & 57 & 23 \\
Atrito pleural & 18 & 3
\end{tabular}

de fatores de risco conhecidos, com ou sem anormalidades radiográficas ou gasométricas, e, se presentes, explicáveis por outro diagnóstico ${ }^{(9)}$.

Porém, para tornar menos subjetiva a estratificação de risco, alguns autores sugerem a realização de um escore para uma determinação mais objetiva do grau de suspeita clínica. Entretanto, deve ser escolhido um escore (Quadro 3) de menor complexidade, para que possa ser realizado numa situação de sala de urgência ${ }^{(30,31,32)}$.

\section{2- Radiografia de tórax}

A radiografia de tórax é, habitualmente, o primeiro método de imagem utilizado durante a elaboração diagnóstica na suspeita de TEP. Além da sua capacidade em evidenciar imagens

\section{5- DIAGNÓSTICO}

Como os achados clínicos de TEP são inespecíficos, torna-se necessária uma investigação diagnóstica complementar, de maior ou menor complexidade, segundo as disponibilidades do local.

Sendo assim, podemos utilizar:

- suspeita clínica (cenário clínico e fatores de risco),

- radiografia de tórax,

- gasometria arterial,

- D-dímeros,

- eletrocardiograma e ecocardiograma,

- cintilografia $\mathrm{V}_{\mathrm{A}} / \mathrm{Q}$,

- tomografia computadorizada helicoidal (angiotomografia),

- arteriografia pulmonar,

- avaliação de membros inferiores (flebografia/ duplex-scan / flebotomografia),

- algoritmos diagnósticos.

\section{1- Suspeita clínica}

Considera-se suspeita clínica de alta probabilidade a identificação de um quadro clínico compatível, em pacientes com fatores de risco definidos para TEV, com ou sem anormalidades radiográficas ou gasométricas associadas, e, se presentes, inexplicáveis por outro diagnóstico.

Suspeita clínica de baixa probabilidade é constituída por um quadro clínico compatível, na ausência compatíveis com o diagnóstico de TEP, pode, muitas vezes, demonstrar a presença de outras alterações, conduzindo a diagnósticos alternativos, como pneumonia, pneumotórax, fratura de arco costal, edema agudo de pulmão e tamponamento pericárdico.

\begin{tabular}{|l|c|}
\hline \multicolumn{2}{|l|}{ Quadro 3 - Modelo para estimar a probabilidade clínica de TEP } \\
\hline Variáveis & $\boldsymbol{N}^{\boldsymbol{o}}$ de Pontos \\
\hline Sinais e sintomas de TVP & 3,0 \\
\hline Outros diagnósticos são menos prováveis que o TEP & 3,0 \\
\hline Frequiência cardíaca > 100 bpm & 1,5 \\
\hline Imobilização ou cirurgia nas últimas quatro semanas & 1,5 \\
\hline Episódio prévio de TEP ou TVP & 1,5 \\
Hemoptise & 1,0 \\
\hline Câncer (atual ou nos últimos seis meses) & 1,0 \\
\hline Probabilidade Clínica & \\
\hline Baixa & $<2,0$ \\
Intermediária & $2,0-6,0$ \\
Alta & $>6,0$ \\
\hline
\end{tabular}

As alterações radiológicas dependem do momento em que foi realizado o exame, a partir da chegada do êmbolo na circulação pulmonar, cuja presença desencadeia diversos distúrbios fisiopatológicos, que podem resultar em alterações estruturais e anatômicas, responsáveis pelas anormalidades radiológicas identificáveis (Quadro 4). 


\begin{tabular}{l|l|}
\hline Quadro 4 - Seqüência de eventos no TEP \\
\hline $\begin{array}{l}\text { Diminuição do surfactante } \\
\text { Perda do surfactante }\end{array}$ & 02 a $03 \mathrm{~h}$ \\
15 a $16 \mathrm{~h}$ \\
\hline $\begin{array}{l}\text { Congestão pulmonar } \\
\text { Hemorragia alveolar }\end{array}$ \\
\begin{tabular}{l} 
Pneumoconstrição \\
\hline $\begin{array}{l}\text { Infarto pulmonar } \\
\text { Resolução da hemorragia } \\
\text { Resolução do infarto }\end{array}$
\end{tabular} \\
\hline
\end{tabular}

Segue a definição de algumas das alterações radiológicas, encontradas no TEP.

- Sinal de Westermark corresponde a uma região de oligoemia (redução do fluxo sangüíneo), associada à amputação e ingurgitamento do ramo da artéria pulmonar, correspondente àquela área.

- Pneumoconstrição é a diminuição volumétrica de uma região pulmonar por redução da atividade local do surfactante. É visualizada como faixas de atelectasias paralelas à cúpula diafragmática, com ou sem a sua elevação.

- O infarto pulmonar costuma apresentar-se sob a forma de uma área de consolidação, grosseiramente triangular e de base voltada para a pleura. Geralmente, está localizado nos lobos pulmonares inferiores. Quando o cone mostra-se arqueado no sentido do hilo, recebe a denominação de Giba ou Corcova de Hampton.

As alterações radiológicas mais freqüentes, no TEP, são as atelectasias e áreas de opacidade no parênquima pulmonar. $\mathrm{O}$ derrame pleural não é tão freqüiente e, quando presente, não ocupa mais do que 1/3 do hemitórax. Outras alterações, como oligoemia (sinal de Westermark), proeminência da artéria pulmonar (sinal de Fleischner), opacidades triangulares, redistribuição vascular e elevação de cúpula diafragmática, mesmo quando presentes, são pobres em predizer o diagnóstico de TEP $(26,32)$.

É importante lembrar que a radiografia de tórax ainda pode ser normal em até 12 - $16 \%$ dos pacientes com diagnóstico confirmado de TEP ${ }^{(26,32)}$.

\section{3- Gasometria arterial}

Os principais achados gasométricos são a hipoxemia $(\mathrm{PaO} 2<80 \mathrm{mmHg})$ e a hipocapnia $(\mathrm{PaCO} 2<35 \mathrm{mmHg}$ ). Os mecanismos envolvidos no desenvolvimento da hipoxemia são as alterações na relação ventilação/perfusão e a redução da pressão parcial de oxigênio no sangue venoso, que chega aos pulmões para ser oxigenado $(\mathrm{PvO} 2)$, em função da diminuição do débito cardíaco. A hipocapnia deve-se à hiperventilação. A diferença alveoloarterial de oxigênio também pode estar aumentada. Seu cálculo está descrito no Quadro 5.

Embora as alterações ocorram na maioria dos pacientes, uma gasometria normal não pode excluir o diagnóstico de TEP, da mesma forma que as alterações encontradas não são específicas para seu diagnóstico ${ }^{(26,27)}$.

Outros fatores que podem prejudicar ainda mais a análise da gasometria arterial, nesses pacientes, são: a oferta de oxigênio antes da sua coleta e/ou a sua realização em pacientes com cardiopatia ou pneumopatia prévias.

\section{Quadro 5 - Cálculo da diferença alvéolo-arterial de oxigênio

$$
\mathrm{PAO} 2=(\mathrm{PB}-47) \times \mathrm{FIO} 2-\mathrm{PaO} 2 / 0,8
$$$$
\mathrm{D}(\mathrm{A}-\mathrm{a}) \mathrm{O} 2=\mathrm{PAO} 2-\mathrm{PaO} 2
$$

Onde: PAO2: pressão alveolar de oxigênio

PaO2: pressão arterial de oxigênio

PB: pressão barométrica

FIO2: fração inspirada de oxigênio

\section{4- D-Dímero}

O D-Dímero (DD) é um fragmento dos produtos de degradação da fibrina (PDF), presentes no sangue, quando o mecanismo de fibrinólise é ativado. Quando há um fenômeno tromboembólico, há ativação do sistema fibrinolítico, elevando as concentrações séricas dos PDF.

Métodos imunológicos são utilizados para detectar a presença de DD no sangue. O primeiro método a ser desenvolvido e rotineiramente utilizado é o teste de aglutinação do látex, um método qualitativo e rápido. O teste quantitativo (ELISA) é mais acurado, porém mais demorado. Há inúmeros kits comercializados para a detecção de DD sérico, cada qual com suas próprias características. São exemplos de testes: SimpleRED, Vidas e MDA D-dimer ${ }^{(9,33)}$. 
De forma geral, apresentam especificidade e valor preditivo positivo, baixos. Por outro lado, possuem sensibilidade alta na presença de embolia pulmonar, onde uma concentração abaixo do cut-off (500ng/ ml) permite afastar tal diagnóstico (dependendo do teste utilizado e levando em conta a probabilidade clínica), com valor preditivo negativo acima de $94 \%{ }^{(34,35)}$.

A dosagem de DD deve ser interpretada sempre em conjunto com a probabilidade clínica. Assim, em pacientes com alta probabilidade clínica, não há necessidade de realizar testes para a detecção de DD, pois nessa situação, nenhum teste será capaz de excluir TEV ${ }^{(9,31)}$.

Um teste negativo para DD (SimpleRED, Vidas, MDA) exclui diagnóstico de TEV em pacientes com baixa probabilidade clínica. Pacientes com probabilidade clínica intermediária necessitam de testes com maior sensibilidade (Vidas, MDA) para excluir esse diagnóstico ${ }^{(9,31)}$.

Há, ainda, situações onde os DDs podem estar aumentados, mesmo na ausência de TEV, constituindo os resultados falso-positivos. São exemplos dessas situações: trauma ou cirurgia nos últimos três meses, inflamação, tumores malignos, CIVD, doença hepática (bilirrubina $>2 \mathrm{mg} / \mathrm{dl}$ ), infecções e septicemia, pré-eclampsia e eclampsia, leucocitose e hematócrito baixo ${ }^{(34)}$.

\section{5- Eletrocardiograma}

O eletrocardiograma (ECG) não é um exame sensível ou específico para o diagnóstico de TEP. Contribuem para seu baixo rendimento a presença de ECG normal, em pacientes com TEP (em até 30\%), a possibilidade de desaparecimento rápido das alterações, dificuldades de interpretação em função de alterações secundárias a doenças cardiopulmonares préexistentes $(26,27,36)$.

As alterações eletrocardiográficas, na fase aguda do TEP, dependem do nível de gravidade da embolia e são, na maioria, inespecíficas. Entre tais alterações, podemos citar: flutter ou fibrilação atrial, onda $P$ pulmonale, desvio do eixo para direita ou esquerda, bloqueio completo ou incompleto de ramo direito, complexos de baixa voltagem, depressão ou elevação do segmento ST e inversão da onda T. A maioria dos pacientes (90\%) com TEP apresenta ritmo sinusal ${ }^{(26)}$.

O padrão S1Q3T3 (presença de onda S em DI, presença de onda Q e inversão da onda T em DIII), descrito como clássico no TEP, não é muito freqüente, ocorrendo em menos de $12 \%$ dos casos ${ }^{(36)}$, predo- minando naqueles de maior gravidade e conseqüentemente associados com disfunção ventricular direita.

Mesmo assim, o ECG tem um papel importante durante a investigação diagnóstica de TEP, pois auxilia na confirmação ou exclusão de importantes diagnósticos diferenciais, como o infarto agudo do miocárdio e a pericardite.

\section{6- Ecocardiograma}

Ainda não há um consenso, na literatura, sobre o real papel do ecocardiograma (ECO) no manejo do TEP, pois pode estar normal em até $45 \%$ dos pacientes com diagnóstico confirmado, ${ }^{(37,38)}$ e há trabalhos, mostrando que ele não é capaz de predizer sobre a mortalidade ${ }^{(39)}$. Outros estudos, porém, mostram que a presença de disfunção ventricular direita, identificada pelo ECO, é um preditor independente de óbito, além de possibilitar medidas seriadas durante a evolução do quadro ${ }^{(40,41,42)}$.

As principais alterações descritas no ECO, em pacientes com diagnóstico de TEP, são: presença de disfunção ventricular direita, moderada ou grave, hipertensão pulmonar, forame oval patente e presença de trombo livre em câmaras cardíacas direitas ${ }^{(42,43)}$.

A razão entre o maior diâmetro diastólico final do ventrículo direito (VD) sobre a mesma medida no ventrículo esquerdo (RVEDA/LVEDA), quando superior a 0,6 , tem sido considerada a melhor forma de identificação e quantificação do distúrbio hemodinâmico $^{(39)}$.

A elevação aguda da pressão pulmonar pelo TEP pode causar isquemia miocárdica regional, com a subseqüente liberação de troponina, que pode ser quantificada. Recentemente, o nível sérico de troponina tem mostrado correlação com a presença de disfunção ventricular direita, e é considerado como um indicador isolado, de pior prognóstico ${ }^{(44,45)}$.

Outro aspecto, que é freqüentemente questionado, é a correlação entre os achados do ECO e a extensão do tromboembolismo. Pacientes com menos de $30 \%$ de comprometimento da circulação pulmonar pelo TEP têm 6,8 vezes mais chances de apresentar um ECO normal, enquanto que, pacientes com 30\% ou mais de comprometimento da circulação pulmonar apresentam, em 92\% dos casos, disfunção de ventrículo direito ${ }^{(46,47)}$.

\section{7- Cintilografia $V_{\mathrm{A}} / \mathbf{Q}$}

A cintilografia pulmonar de ventilação e perfusão é considerada um dos exames diagnósticos mais 
importantes em embolia pulmonar. Como o método é baseado na injeção, em veia periférica, de macroagregado marcado com material radioativo, a ausência de captação em determinada região é prova incontestável de ausência de fluxo sangüíneo. Isso ocorre não só na embolia pulmonar, mas, também, em outras condições, como massas tumorais, lesões cavitárias e ressecções pulmonares. O radiofármaco utilizado, atualmente, é o tecnécio. O mapeamento de ventilação é obtido com a inalação de partículas radioativas, também marcadas com tecnécio. Os distúrbios não pareados, com perfusão ausente e ventilação presente, caracterizam a embolia pulmonar. Devido à dificuldade de interpretação dos mapeamentos, vários autores se preocuparam em padronizá-la. A interpretação dos achados cintilográficos em TEP, derivados do Prospective Investigation of Pulmonary Embolism Detection (PIOPED), estão ilustrados no Quadro 6 e seus resultados foram aferidos por análise de arteriografias pulmonares, realizadas na população estudada, e a probabilidade clínica estimada foi incorporada à sua interpretação ${ }^{(25)}$.

Assim, nos casos de alta probabilidade clínica, cuja cintilografia $\mathrm{V}_{\mathrm{A}} / \mathrm{Q}$ é de alta probabilidade, a possibilidade diagnóstica de TEP é de $96 \%$. No outro extremo, em casos de baixa probabilidade clínica, em que a cintilografia $\mathrm{V}_{\mathrm{A}} / \mathrm{Q}$ é de baixa probabilidade ou é normal, o diagnóstico de TEP é muito improvável, sendo de apenas $2-4 \%$. Todas as outras possibilidades tornam o resultado inconclusivo, o que costuma ocorrer em 2/3 dos casos. Na presença de insuficiência cardíaca ou de DPOC, a possibilidade de que a cintilografia pulmonar traga resultados definitivos é ainda menor ${ }^{(25)}$.

$\mathrm{Na}$ ausência da cintilografia de ventilação, a cintilografia de perfusão deve ser comparada com a radiografia de tórax. Preconiza-se que, sendo normal a cintilografia de perfusão, não há necessidade de se realizar a cintilografia de ventilação. Se a cintilografia de perfusão mostrar alterações perfusionais segmentares, em regiões normais à radiografia de tórax, assume-se a alta probabilidade para TEP e, mais uma vez, não há necessidade de realizar a cintilografia de ventilação. Porém, se este exame for realizado em pacientes com doenças cardiopulmonares prévias, indica-se que se realize a cintilografia de perfusão e a de ventilação ${ }^{(35)}$.

Atualmente, a cintilografia $\mathrm{V}_{\mathrm{A}} / \mathrm{Q}$ continua tendo importante papel no diagnóstico de TEP. Entretanto, alguns aspectos devem ser salientados. Primeiro, ela deve ser realizada em pacientes sem alterações cardiopulmonares prévias, segundo, um resultado normal tem a capacidade de excluir o diagnóstico de TEP; por outro lado, uma cintilografia de alta probabilidade não é capaz de, sozinha, confirmar tal diagnóstico ${ }^{(9,25,31)}$.

\section{8- Tomografia computadorizada helicoidal}

A utilização da tomografia computadorizada, na investigação de TEP, pode ser considerada um dos maiores avanços nessa área. Além de possibilitar a visualização direta do trombo, ela permite a identificação de alterações no parênquima pulmonar. Com isso, ela pode contribuir para o diagnóstico de TEP ou mostrar outras possibilidades diagnósticas.

Para a tomografia helicoidal, as taxas de sensibilidade estão entre 57 a $100 \%$, com especificidade de 78 a $100 \%{ }^{(48,49)}$. Na detecção de êmbolos localizados em artérias subsegmentares, a sensibilidade cai para 71 a $84 \%{ }^{(50)}$, porém, o tromboembolismo isolado desses ramos não é usual, ocorrendo em 6 a 30\% dos pacientes com TEP, em diferentes séries estudadas ${ }^{(51,52)}$. Atualmente, com o uso de novas técnicas, a visualização das artérias segmentares e subsegmentares tem aumentado e melhorado, ainda mais, a concordância entre vários examinadores ${ }^{(53)}$.

Quadro 6 - Prospective Investigation of Pulmonary Embolism Detection (PIOPED)

\begin{tabular}{|c|c|c|c|c|c|}
\hline \multirow{2}{*}{ Cintilografia $\mathbf{V / Q}$} & \multicolumn{2}{|c|}{ Probabilidade Clínica Estimada (\%) } & Total & N \\
\hline Alta & $\mathbf{8 0 - 1 0 0}$ & $\mathbf{2 0 - 7 9}$ & $\mathbf{0 - 1 9}$ & & 118 \\
\hline Intermediária & 96 & 88 & 56 & 87 & 345 \\
\hline Baixa & 66 & 28 & 16 & 30 & 296 \\
\hline Normal & 40 & 16 & 4 & 14 & 128 \\
\hline
\end{tabular}


Entretanto, uma cintilografia normal exclui o diagnóstico de TEP, o mesmo não acontecendo com a tomografia $(31,35,54,55)$.

\section{9- Arteriografia pulmonar}

É o método ainda considerado como o padrão-ouro, no diagnóstico de TEP. Porém, a inacurácia revelada em alguns estudos, principalmente no que se refere à variabilidade de interpretação entre observadores independentes, e o seu elevado custo sugerem a necessidade de outras estratégias diagnósticas. Entretanto, apesar do caráter invasivo, é um método seguro ${ }^{(56,57)}$, embora pouco utilizado na prática clínica.

\subsection{0- Avaliação de membros inferiores}

A duplex ultrassonografia é positiva em 10 a $20 \%$ dos pacientes sem sinais ou sintomas de TVP, e em aproximadamente $50 \%$ nos pacientes com TEP confirmado ${ }^{(58)}$.

Tem grande papel na investigação de TEV, pois um resultado positivo, em um paciente com suspeita clínica de TEP, que apresente alguma alteração de membro inferior que sugira TVP, é suficiente para a conclusão diagnóstica.

Entretanto, um exame normal não exclui o diagnóstico de TEP. Por outro lado, um resultado positivo, em um paciente sem sinais ou sintomas de membros inferiores, deve ser interpretado com cautela ${ }^{(59)}$. A presença de um ou mais dos fatores relacionados no Quadro 7, aumenta a chance de resultados falso$\operatorname{positivos}^{(58)}$.

Quadro 7 - Fatores que diminuem o valor preditivo, positivo, do US de membros inferiores, em pacientes com TEP agudo

- Paciente assintomático para TVP

Baixa suspeita clínica de TVP

Alteração restrita a um pequeno segmento de veia proximal

Anormalidade restrita às veias da panturrilha

Episódio prévio de TVP

Resultado negativo de teste sensível para TVP (exemplo: dosagem de D-dímeros)

Adaptado de Kearon C et al., $1998^{(58)}$
Assim, a venografia continua sedo o padrão-ouro para a avaliação de membros inferiores e tem importante papel, quando se avalia esse grupo de pacientes ${ }^{(58,60)}$.

A flebotomografia tem sido incorporada ao arsenal de exames para o diagnóstico de TVP, porém não de forma independente. Com o paciente mantido na mesma posição e utilizando o mesmo contraste injetado por bomba em veia periférica, visando à pesquisa de TEP (angiotomografia pulmonar), são realizados cortes de espessuras variáveis, conforme o protocolo preconizado na instituição, desde a cavidade pélvica até a extremidade distal das pernas. Com a flebotomografia, haveria a vantagem de avaliar pulmões e membros inferiores com um único exame e visualizar sítios de trombose que não estariam no campo de avaliação da ultra-sonografia. Assim, tal método tem sido elaborado cada vez mais, com o objetivo de diminuir o tempo do exame e a dose de radiação. Os resultados, na literatura, ainda não são uniformes, porém alguns dados são promissores ${ }^{(61)}$.

\subsection{1- Algoritmos diagnósticos}

Fundamentados nas diversas informações, vários algoritmos diagnósticos têm sido formulados. As Figuras de 4 a 7 mostram alguns dos algoritmos propostos mais recentemente, baseados, inicialmente, na suspeita clínica. ${ }^{(31)}$.

Pacientes com doença pulmonar prévia (parenquimatosa ou de via aérea) ou pacientes com outros diagnósticos diferenciais devem realizar, preferencialmente, a angiotomografia pulmonar, pois, nestes casos, a cintilografia V/Q pode não auxiliar muito no diagnóstico de TEP ${ }^{(62,63)}$.

Pacientes com TEP podem permanecer com alterações perfusionais mesmo após três meses de tratamento. Dessa forma, na suspeita de recidiva de TEP, a arteriografia é o exame de escolha, pois consegue diferenciar as alterações crônicas de um novo episódio tromboembólico ${ }^{(31,64)}$.

Em pacientes com insuficiência renal, a angiotomografia deve ser evitada e a investigação diagnóstica deve ser feita com cintilografia V/Q e ultrasonografia de membros inferiores. Se esses exames não forem suficientes para firmar o diagnóstico de TEP, dever-se-á realizar a arteriografia pulmonar ${ }^{(65)}$.

Além disso, em pacientes instáveis, é preferível o uso de métodos que tragam resultados rápidos e definitivos, como a arteriografia pulmonar. Já, nos pacientes em ventilação mecânica, a ultra-sonografia de membros inferiores deve ser o primeiro exame a ser realizado $^{(31)}$. 


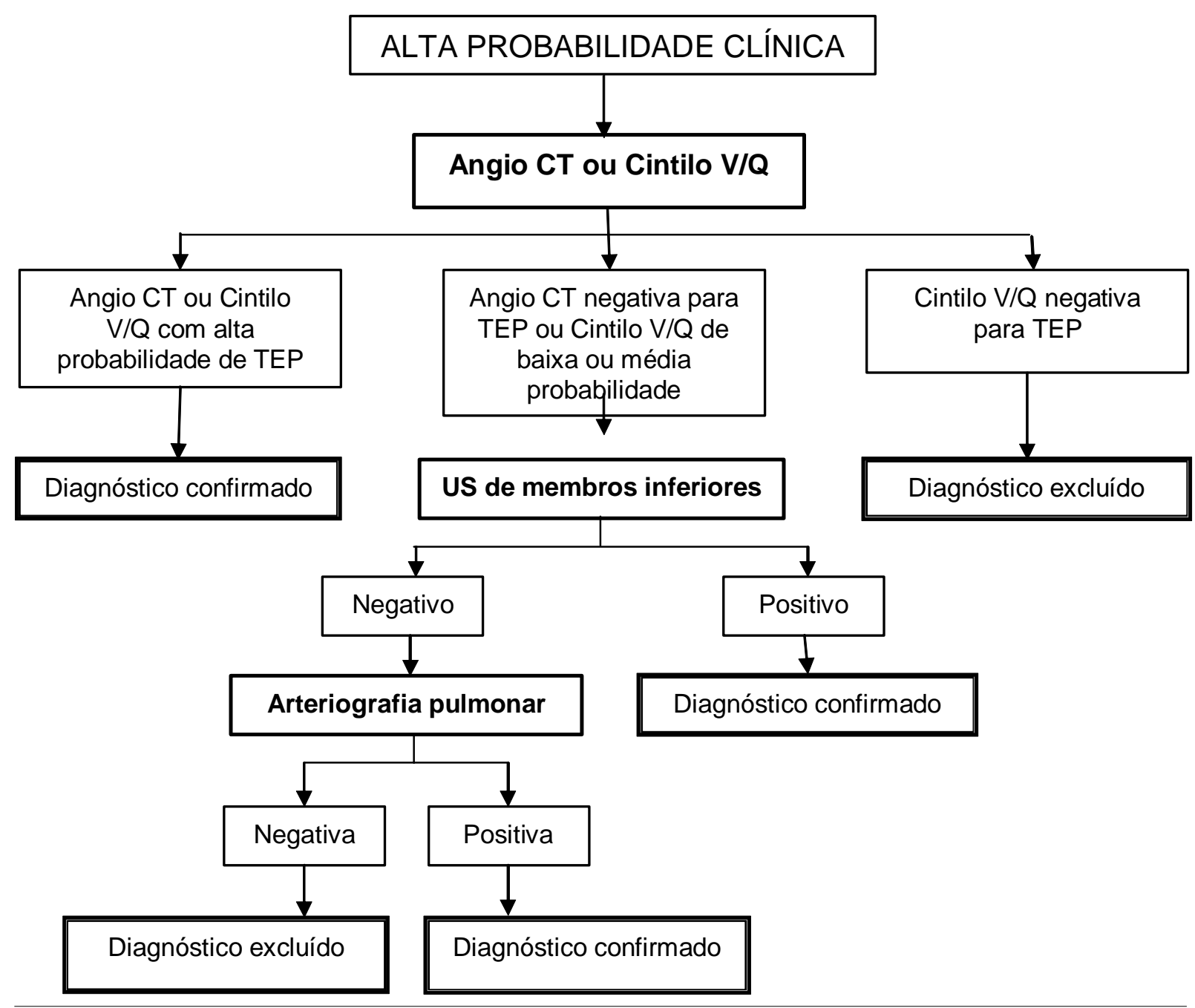

Figura 4 - Algoritmo diagnóstico em pacientes com alta probabilidade clínica para TEP, utilizando, inicialmente, Angio CT ou Cintilo V/Q. Fedullo PF et al., $2003^{(31)}$.

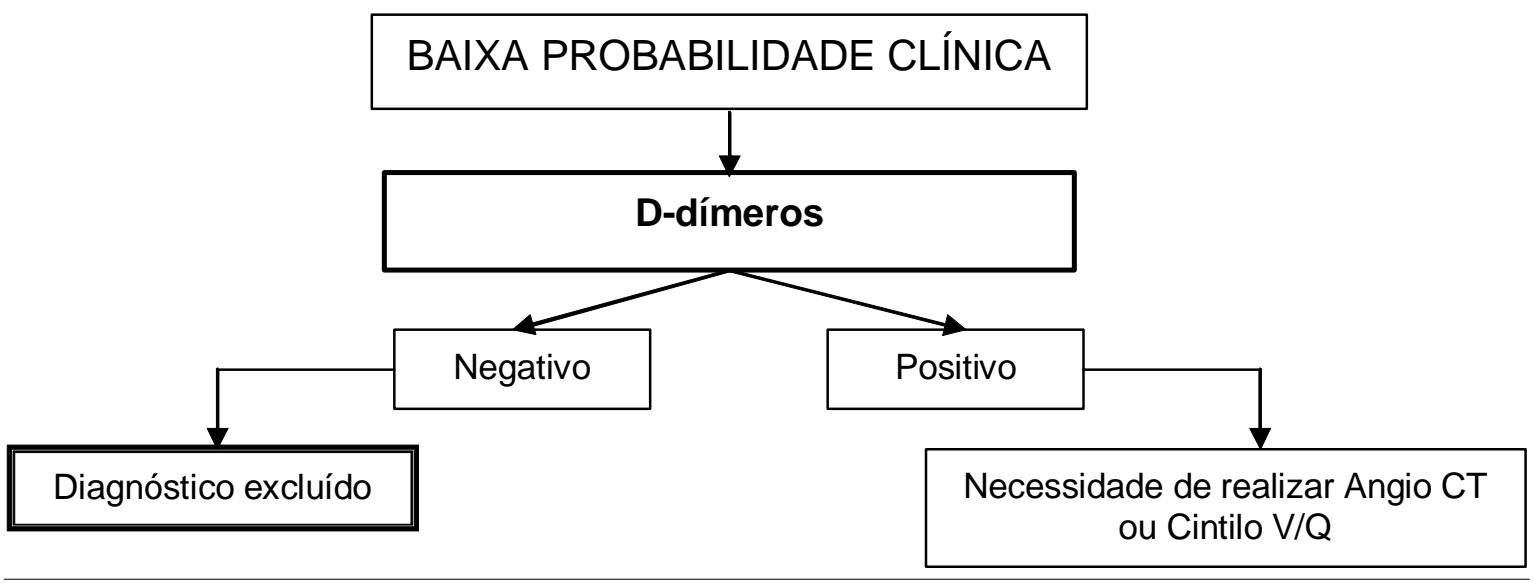

Figura 5 - Algoritmo diagnóstico em pacientes com baixa probabilidade clínica para TEP, utilizando inicialmente D-dímeros. Fedullo PF et al, $2003^{(31)}$. 


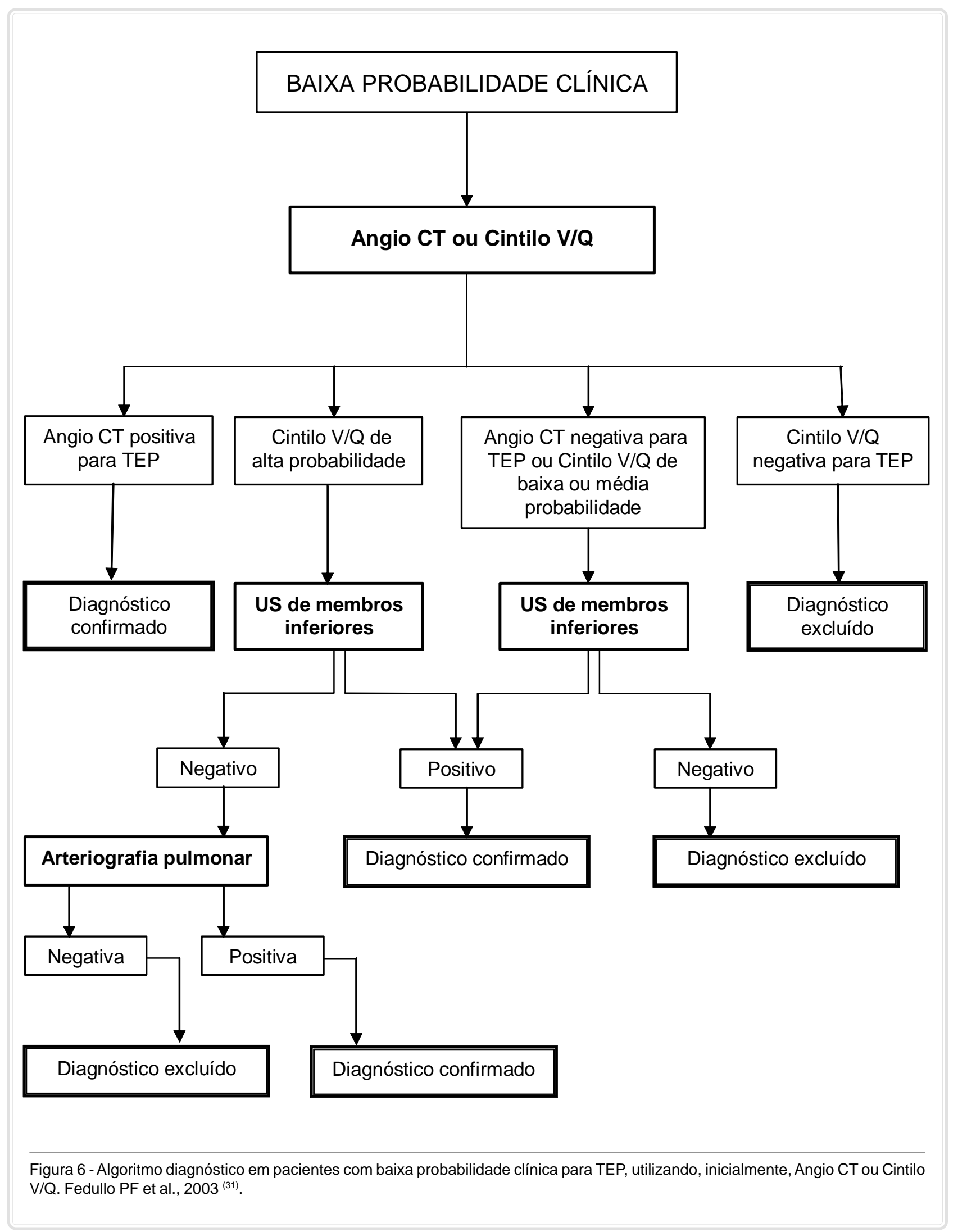




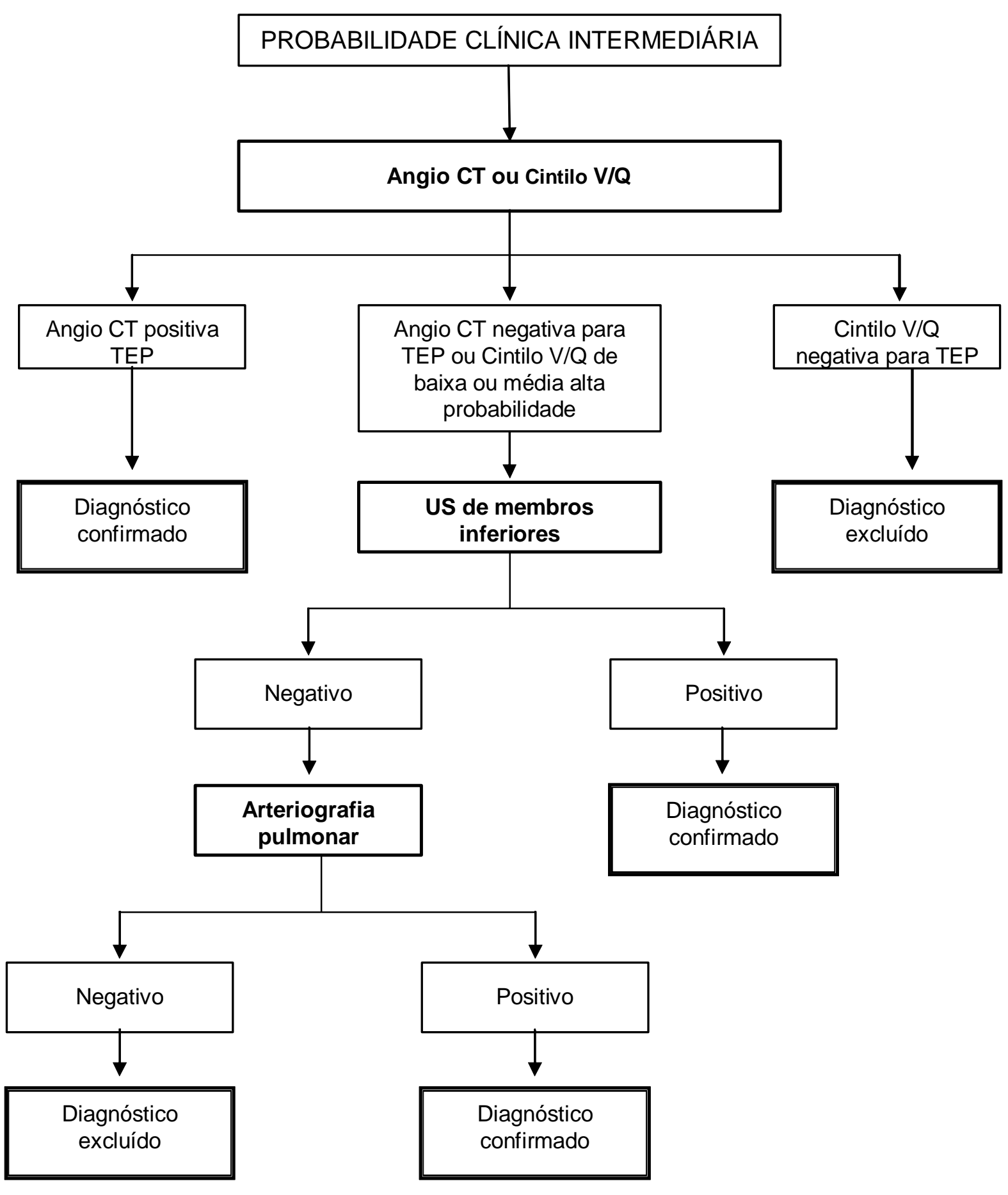

Figura 7 - Algoritmo diagnóstico em pacientes com probabilidade clínica intermediária para TEP, utilizando, inicialmente, Angio CT ou Cintilo V/Q. Fedullo PF et al, $2003^{(31) .}$ 
$\mathrm{Na}$ ausência de exames complementares mais sofisticados, a suspeita clínica (baseada nos sinais, sintomas e fatores de risco) assume papel fundamental, e a investigação disponível será realizada com o objetivo principal de excluir outros diagnósticos, e não com o objetivo de confirmar o TEP. Se um outro diagnóstico não for estabelecido (pneumotórax, infarto agudo do miocárdio, edema pulmonar, pneumonia, entre outros), dever-se-á iniciar o tratamento para TEP, mesmo sabendo que alguns pacientes serão tratados sem que, realmente, tenham o diagnóstico correto. Entretanto, em determinadas situações, mais de um tratamento deverá ser instituído, em função da impossibilidade de se fazer um diagnóstico de certeza. Nestes casos, a avaliação seqüencial e evolutiva do paciente é fundamental e indispensável, pois permite o esclarecimento diagnóstico em um segundo momento.

No entanto, a aplicação desses algoritmos irá depender dos recursos disponíveis e da experiência de cada serviço.

\section{6- TRATAMENTO}

\section{1- Medidas gerais de suporte}

O paciente deve ser mantido em ambiente hospitalar.

Como o pulmão possui três fontes possíveis de suprimento de oxigênio para seus tecidos (artérias pulmonares, vias aéreas e artérias brônquicas), deverá sempre ser fornecido oxigênio suplementar para os pacientes com TEP. Analgesia pode ser utilizada, quando houver necessidade.

\section{2- Heparina não fracionada (HNF)}

A administração da heparina deve ser endovenosa, com uma dose de ataque seguida da manutenção por infusão contínua. Para a anticoagulação venosa contínua, são dois os esquemas possíveis de administração, um com dose baseada no peso do paciente e outro com dose empírica, conforme ilustrado nos Quadros 8 e $9^{(66)}$. Qualquer que seja o esquema adotado, ele será baseado nos resultados do TTPA (tempo de tromboplastina parcial ativada), pois a heparina padrão (ou não fracionada) apresenta características farmacológicas, que tornam imprevisíveis os seus resultados em diferentes pacientes, ou seja, a administração de uma dose fixa de heparina não garante a anticoagulação. $\mathrm{O}$ esquema com dose baseada no peso do paciente mostrou-se mais efetivo e seguro ${ }^{(66)}$.

A heparina deve ser mantida por um mínimo de cinco dias e não deve ser suspensa até que níveis adequados de anticoagulação oral tenham sido atingidos (INR > 2,0). O anticoagulante oral deve ser iniciado simultaneamente, devendo ter um período de sobreposição de 04 a 05 dias. Em pacientes com TEP maciço ou com extensa trombose íleofemoral, a anticoagulação com heparina deve se estender por 10 dias ${ }^{(67)}$.

Sugestão de preparo da solução de heparina para infusão contínua:

$$
\left\{\begin{array}{l}
\text { Soro glicosado } 5 \%-250 \mathrm{ml} \\
\text { Heparina-padrão }-5 \mathrm{ml}(25.000 \mathrm{U}) \\
1 \mathrm{ml}=20 \text { gotas }=60 \text { microgotas }=100 \mathrm{U}
\end{array}\right.
$$

\section{Quadro 8 - Heparinização com dose baseada no peso do paciente}

Dose inicial $\quad 80 \mathrm{U} / \mathrm{Kg}$, endovenosa

Manutenção Infusão contínua de $18 \mathrm{U} / \mathrm{Kg} / \mathrm{h}$ e ajuste da dose, segundo resultado de TTPA colhido a cada 6 h*.

TTPA $<1,2 \quad$ Novo bolus de $80 \mathrm{U} / \mathrm{Kg}$ e aumento da infusão contínua em $4 \mathrm{U} / \mathrm{Kg} / \mathrm{h}$

1,2 $\leq$ TTPA $\leq 1,5$ Novo bolus de $40 \mathrm{U} / \mathrm{Kg} / \mathrm{h}$ e aumento da infusão contínua em $2 \mathrm{U} / \mathrm{Kg} / \mathrm{h}$

$1,5<$ TTPA $\leq 2,3$ Manter a infusão sem alterações

$2,3<$ TTPA $\leq 3,0$ Redução da infusão contínua em $2 \mathrm{U} / \mathrm{Kg} / \mathrm{h}$

TTPA > 3,0 Interrupção da infusão contínua por $1 \mathrm{~h}$, seguida da redução da infusão contínua em $3 \mathrm{U} / \mathrm{Kg} / \mathrm{h}$

* Após dois resultados de TTPA, na faixa terapêutica, o exame passa a ser realizado a cada $24 \mathrm{~h}$. Se o TTPA sair da faixa terapêutica,

retorna-se ao esquema inicial. 


\begin{tabular}{|c|c|}
\hline \multicolumn{2}{|c|}{ Quadro 9 - Heparinização com dose empírica } \\
\hline Dose inicial & $5000 \mathrm{U}$, endovenosa \\
\hline Manutenção & Infusão contínua de 1000 U/h e ajuste da dose, segundo resultado de TTPA colhido a cada 6 h*. \\
\hline TTPA $<1,2$ & Novo bolus de 5000 U e aumento da infusão contínua em 200 U/h \\
\hline $1,2 \leq \mathrm{TTPA} \leq 1,5$ & Novo bolus de 2500 U e aumento da infusão contínua em 100 U/h \\
\hline $1,5<$ TTPA $\leq 2,3$ & Manter a infusão sem alterações \\
\hline $2,3<\mathrm{TTPA} \leq 3,0$ & Redução da infusão contínua em 100 U/h \\
\hline TTPA $>3,0$ & Interrupção da infusão contínua por 1 h, seguida da redução da infusão contínua em 200 U/h \\
\hline
\end{tabular}

Uma das complicações do tratamento com heparina é o sangramento. Sua freqüência varia de 0 a $7 \%$. A freqüência de sangramento fatal é de 0 a $2 \%$. Esse risco aumenta em função da dose de heparina utilizada e da idade do paciente ${ }^{(68)}$.

Outra complicação é a trombocitopenia, ocorrendo em menos de $1 \%$ dos pacientes, quando a heparina é administrada por não mais do que 05 a 07 dias. Geralmente, ocorre entre o sétimo e o décimo quarto dia de uso da heparina. Sendo assim, é aconselhável a contagem de plaquetas em três tempos: entre o $3^{\circ}$ e o $5^{\circ}$ dia, entre o $7^{\circ}$ e o $10^{\circ}$ dia e no $14^{\circ}$ dia. Deve ser interrompido seu uso, quando a contagem de plaquetas atingir níveis abaixo de $100.000 / \mathrm{mm}^{3}$ ou quando ocorrer uma queda de $50 \%$ do valor inicial ${ }^{(66)}$. Essa síndrome é mediada imunologicamente e pode estar associada com tromboembolismo arterial e extensão ou

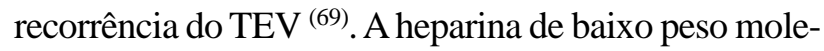
cular tem reação cruzada com a heparina, em até $90 \%$ dos casos (in vitro) e, portanto, não deve ser usada na fase aguda da complicação. No tratamento da trombocitopenia induzida pela heparina e associada à trombose, temos: danaparoide, hirudina recombinante, plasmaferese e IgE imune. Tal quadro não deve ser confundido com a trombocitopenia moderada, transitória, que ocorre frequientemente nos primeiros dias de terapia com heparina e não necessita de nenhuma intervenção.

A osteoporose pode ocorrer com o uso prolongado da heparina (acima de três meses) e sua incidência e severidade é proporcional à dose e à duração do tratamento. Conforme ocorre perda progressiva da densidade óssea, o processo torna-se menos reversível. Nenhuma terapia preventiva foi comprovada para diminuir esse risco, porém suplementos de vitamina $\mathrm{D}$ e cálcio podem ser oferecidos, principalmente para gestantes.

Outras complicações mais raras são: alterações discretas na função hepática (elevações de transaminases, bilirrubinas e fosfatase alcalina) ${ }^{(70)}$, hipercalemia (em alguns pacientes com hiperaldosteronismo) $)^{(71)}$ e diminuição dos níveis de antitrombina III (o que causar trombose generalizada) ${ }^{(72)}$.

Os efeitos da heparina administrada endovenosamente desaparecem em poucas horas, após sua suspensão. Sendo assim, sangramentos leves e superficiais não requerem tratamentos especiais a não ser a sua suspensão. Nos sangramentos mais graves, a reversão da heparinização é feita com sulfato de protamina. Cada $1 \mathrm{mg}$ de protamina reverte $100 \mathrm{U}$ de heparina. A dose usual não deve exceder $50 \mathrm{mg}$ de protamina, e deve ser administrada em infusão venosa durante 10 a $20 \mathrm{~min}$. Quando em infusão, apenas a heparina administrada na última hora deve ser incluída no cálculo da dose a ser neutralizada, pois a meia-vida da heparina não fracionada, quando administrada endovenosamente, é de, aproximadamente, $60 \mathrm{~min}$. Os efeitos colaterais da protamina são raros e incluem: hipotensão e bradicardia (que podem ser minimizadas com a infusão mais lenta da protamina) e reações alérgicas, incluindo anafilaxia (mais comum em pacientes que recebem insulina, vasectomizados ou com antecedente de alergia a peixe). Pacientes com risco de apresentar anticorpos antiprotamínicos devem ser previamente tratados com corticóides e anti-histamínicos ${ }^{(73)}$. 


\section{3- Heparina de baixo peso molecular (HBPM)}

Os trabalhos de revisão mais atuais recomendam que as HBPM devem ser usadas não só na profilaxia, mas, também, como tratamento nos casos de $\operatorname{TEV}(9,67)$.

As HBPM têm peso molecular de aproximadamente $1 / 3$ do peso da heparina não-fracionada, com peso médio entre 4000 e 5000d. Por elas apresentarem uma menor ligação celular e proteica, oferecem efeitos biológicos vantajosos, como ilustrado no Quadro 10.

Sua resposta anticoagulante (mensurada pelo nível plasmático de anti Xa U/ml) é correlacionada com o peso corporal e a HBPM é efetiva na maioria dos pacientes, quando administrada com base no peso corporal (anti Xa U/Kg) sem a necessidade de posterior monitorização laboratorial ou ajuste de dose ${ }^{(74)}$. Há, no entanto, algumas situações clínicas que justificam a monitorização da dose da HBPM: pacientes com clearance de creatinina $<30 \mathrm{ml} / \mathrm{min}^{(67)}$, uma vez que essa heparina também tem excreção renal; gestantes, pelo possível aumento do clearance da heparina; obesos (IMC > 30), pois ainda não há estudos com esse grupo de pacientes; e ainda, pacientes idosos ou com maior risco de sangramento ${ }^{(75,76,77)}$. A forma preconizada para o acompanhamento da anticoagulação com HBPM é aquela através da dosagem plasmática do anti Xa, que deve ser realizada quatro horas após sua administração subcutânea. Para os esquemas terapêuticos com dose fracionada (duas vezes ao dia), o nível sérico do anti Xa deve estar entre 0,6 a 1,0 U/ml. Para os esquemas com dose única diária, o valor sérico do anti Xa deve estar entre 1,0 a 2,0 U/ml ${ }^{(76,77)}$.

A protamina não neutraliza completamente a atividade do antiXa e novas drogas com tal ação, ainda estão em estudo ${ }^{(78,79)}$. Enquanto isso, nos casos em que há necessidade de reverter a anticoagulação pela HBPM, preconiza-se o seguinte esquema: dentro das primeiras oito horas, após a administração da heparina, $1 \mathrm{mg}$ de protamina para cada $1 \mathrm{mg}$ de enoxaparina ou para cada $100 \mathrm{U}$ de dalteparina. Se o sangramento persistir, uma segunda dose será necessária, porém, agora, na proporção de $0,5 \mathrm{mg}$ de protamina para cada $1 \mathrm{mg}$ de enoxaparina. Doses menores podem ser necessárias após oito horas da administração da HBPM ${ }^{(67)}$.

As heparinas de baixo peso molecular de que dispomos, no momento, são a nadroparina, a enoxaparina e a dalteparina. A posologia de cada uma delas está ilustrada no Quadro 11 e a duração do tratamento é a mesma preconizada para a heparina não fracionada.

\section{4- Heparina não fracionada $x$ HBPM}

Ainda não há, na literatura, um consenso sobre qual é a melhor heparina no tratamento do TEV e, por enquanto, elas são consideradas equivalentes ${ }^{(67)}$.

Apesar de alguns trabalhos ${ }^{(80,81)}$ já terem demonstrado um benefício no uso das HBPMs em pacientes com câncer, reduzindo a mortalidade das pessoas desse grupo, o assunto ainda é exploratório. Em alguns estudos animais, a administração de HBPM diminuiu a incidência de metástases ${ }^{(82)} \mathrm{e}$ a angiogênese necessária para o crescimento tumoral ${ }^{(83)}$.

\section{5- Hirudina}

A hirudina é um peptídeo que inibe a trombina de forma direta, independentemente da sua interação com a antitrombina. É uma droga promissora no tratamento e na profilaxia de TEV, em especial nos casos com reação alérgica ao uso da heparina ${ }^{\left({ }^{(8)}\right.}$.

\section{6- Anticoagulante oral}

O anticoagulante oral mais utilizado, no tratamento da TEV/TEP, é a warfarina. Entre suas características farmacocinéticas temos que o pico plas-

\section{Quadro 10 - Características das HBPM}

\begin{tabular}{|l|l|}
\hline Alvo & Efeito Biológico \\
\hline Trombina & anti IIa / anti Xa reduzida \\
\hline Proteínas & resposta anticoagulante mais previsível \\
\hline Macrófagos & depuração renal \\
\hline Plaquetas & menor indução de anticorpos \\
\hline Osteoblastos & menor ativação de osteoclastos \\
\hline
\end{tabular}

\section{Conseqüiência}

desconhecida

monitoramento desnecessário

meia-vida mais longa, possibilitando dose única, diária

menor risco de trombocitopenia

menor incidência de osteopenia 


\begin{tabular}{|c|c|}
\hline \multicolumn{2}{|c|}{$\begin{array}{l}\text { Quadro } 11 \text { - Posologia das HBPM no tratamento da } \\
\text { TEV }\end{array}$} \\
\hline Heparina & Posologia \\
\hline $\begin{array}{l}\text { Nadroparina } \\
\text { (Fraxiparina } ®)\end{array}$ & $\begin{array}{l}450 \mathrm{U} / \mathrm{Kg} / \mathrm{dia} \text {, subcutânea, } \\
\text { administrada de } 12-12 \mathrm{~h} \\
(\text { cada } 0,3 \mathrm{cc}=3075 \mathrm{U})\end{array}$ \\
\hline Enoxaparina (Clexane®) & $\begin{array}{l}2 \mathrm{mg} / \mathrm{Kg} / \mathrm{dia} \text {, subcutânea, } \\
\text { administrada de } 12-12 \mathrm{~h} \\
\text { não ultrapassar } 180 \mathrm{mg} / \mathrm{dia}\end{array}$ \\
\hline Dalteparina (Fragmin®) & $\begin{array}{l}200 \text { U/Kg/dia, subcutânea, } \\
\text { administrada de } 12-12 \mathrm{~h} \\
\text { não ultrapassar } 18000 \mathrm{U} / \text { dia }\end{array}$ \\
\hline
\end{tabular}

mático ocorre após 90 min da sua administração oral e sua meia-vida é de 36 a 42 h. Sua ação anticoagulante é secundária à inibição da síntese dos fatores da coagulação, dependentes de vitamina K (fatores II, VII, IX e X). Assim, seu efeito só surge, quando ocorre, na circulação, o desaparecimento desses fatores, já previamente sintetizados, o que, geralmente, acontece em 2 a 7 dias, dependendo da dose administrada ${ }^{(85,86)}$.

A dose de warfarina é ajustada pelo tempo de protrombina, cujo resultado é normatizado sob a forma de INR. Pode ser iniciada no primeiro dia de tratamento, na dose diária de $5 \mathrm{mg}$ (um comprimido). A dose inicial preconizada é de $5 \mathrm{mg}$ por dia, entretanto, em pacientes idosos, desnutridos, portadores de doença hepática ou com alto risco de sangramento, a dose inicial pode ser menor.

Em pacientes instáveis ou com possibilidade de serem submetidos a procedimentos invasivos (traqueostomias, punções venosas profundas, inserções de cateteres, biópsias ou cirurgias), o uso do anticoagulante oral deve ser adiado, pois a reversão da sua anticoagulação é mais difícil do que a promovida pela heparina.

A heparina pode ser descontinuada após o INR ter atingido a faixa desejada, em, pelo menos, duas medidas consecutivas com intervalo de $24 \mathrm{~h}$.

O medo de a warfarina criar um estado prótrombótico, quando administrada isoladamente, na fase inicial do tratamento de TVP, em pacientes com deficiência de proteína $C$ (e que, portanto, não estão recebendo, simultaneamente, heparina) não tem sido substanciado. Entretanto, em pacientes com deficiência de proteína $\mathrm{C}$ ou outra forma de trombofilia, julgase prudente a administração concomitante do anticoagulante oral e da heparina ${ }^{(67)}$.
O INR deve ser mantido entre 02 e 03 . Os pacientes com síndrome do anticorpo antifosfolípide são de manejo clínico mais difícil, podendo ocorrer recidivas de fenômenos trombembólicos mesmo naqueles com níveis de anticoagulação na faixa terapêutica; mesmo assim, esses pacientes devem ser mantidos com INR entre 02 e $03^{(87)}$.

A monitorização da anticoagulação com o INR deve ser realizada diariamente, até atingir o intervalo terapêutico, e tal medida deve ser mantida por, pelo menos, dois dias consecutivos, quando passa a ser usada duas a três vezes por semana, durante uma ou duas semanas. Após, deve ser usada a cada quatro semanas, no máximo ${ }^{(67)}$. Se houver necessidade de ajuste de dose, deve-se aumentar ou diminuir $20 \%$ da dose semanal e, novo INR deve ser solicitado em uma semana. No paciente que apresentar INR entre 1,8 e 2,0 ou entre 3,1-3,4, deve-se manter a dose do anticoagulante, após ser investigada a dieta e uso correto da medicação, então solicitar novo INR em uma semana. Se o valor do INR permanecer dentro das faixas citadas acima, só então é que deverá ser feita a correção em $20 \%$ da dose do anticoagulante.

É fundamental orientar o paciente a manter sempre, na dieta, a mesma quantidade de verduras verdes, de forma que a alimentação não cause variações significativas de INR.

Outro aspecto importante é que algumas drogas podem influenciar a farmacocinética da warfarina pela inibição da síntese ou pelo aumento do clearance de fatores da coagulação dependente de vitamina $K$ ou, interferindo com outras vias da coagulação (Quadro 12). O ácido acetilsalicílico (AAS), os antinflamatórios não esteroidais (AINE) e elevadas doses de penicilina aumentam o risco de sangramento, associado ao uso de anticoagulante oral, por inibirem a função plaquetária ${ }^{(88,89)}$. Além disso, tanto o AAS como os AINEs podem causar lesão gástrica e, conseqüentemente, hemorragia digestiva.

O sangramento é a principal complicação do uso da warfarina, estando associado com o nível do INR, história prévia de sangramento (principalmente do trato gastrointestinal), antecedentes de AVC, HAS, insuficiência renal e anemia ${ }^{(90,91)}$. Ainda não está estabelecido se a idade do paciente é um fator de risco independente.

Outra complicação, menos freqüente, é a necrose de pele, que, geralmente, ocorre entre o terceiro e o oitavo dia e é mais comum entre pacientes com deficiência de proteínas $\mathrm{C}$ ou $\mathrm{S}^{(92,93)}$. Esse fenômeno é conseqüência de extensa trombose das vênulas e capilares da gordura do subcutâneo. O tratamento dessa 


\begin{tabular}{|c|c|c|c|}
\hline \multicolumn{4}{|c|}{ Quadro 12 - Interação da warfarina com outras drogas } \\
\hline \multicolumn{2}{|c|}{ Potencializa } & \multirow{2}{*}{\begin{tabular}{|l|}
\multicolumn{1}{|c|}{ Inibe } \\
Barbitúricos
\end{tabular}} & \multirow{2}{*}{$\begin{array}{l}\text { Sem interação } \\
\text { Álcool }\end{array}$} \\
\hline Isoniazida & Metronidazol & & \\
\hline Amiodarona & Dissulfiram & Carbamazepina & Hidróxido de alumínio \\
\hline Esteróides anabolizantes & Fenilbutazona & Clordiazepóxido & Atenolol \\
\hline Cimetidina & Piroxican & Colestiramina & Bumetadina \\
\hline Omeprazol & Propafenona & Griseofulvina & Enoxacin \\
\hline Clofibrato & Propranolol & Nafcilina & Famotidina \\
\hline Cotrimoxazol & Acetaminofen & Rifampicina & Fluoxetina \\
\hline Eritromicina & Hidrato de cloral & Sucralfato & Metoprolol \\
\hline Ciprofloxacina & Fenitoína & Dicloxacilina & Naproxen \\
\hline $\begin{array}{l}\text { Álcool em pacientes com } \\
\text { doença hepática }\end{array}$ & Tamoxifeno & $\begin{array}{l}\text { Alimentos com alto teor de } \\
\text { vitamina } K\end{array}$ & Psyllium \\
\hline Itraconazol & Tetraciclina & & Ranitidina \\
\hline Miconazol & Vacina da gripe & & Ibuprofeno \\
\hline Fluconazol & & & Cetoconazol \\
\hline
\end{tabular}

complicação é difícil, e sugere-se que a warfarina seja suspensa e o paciente volte a ser anticoagulado com heparina. Após a melhora do quadro, a warfarina pode ser reintroduzida, porém em doses baixas $(2 \mathrm{mg}$ ) e com aumento progressivo ao longo de semanas.

A administração de vitamina $\mathrm{K}$ pode reverter a anticoagulação decorrente do uso da warfarina. Pacientes com valores elevados de INR devem receber uma dose de vitamina $\mathrm{K}$ suficiente para restaurar o INR dentro da faixa de segurança, mas não subterapêutica, o que poderia causar resistência, por até uma semana, quando fosse reiniciada a terapia com anticoagulante oral.

A vitamina $\mathrm{K}$ pode ser administrada via endovenosa, subcutânea ou oral. A forma endovenosa deve ser utilizada apenas para casos com urgência de reversão da anticoagulação, pois pode ocorrer reação anafilática durante sua infusão. A administração subcutânea proporciona uma resposta imprevisível e demorada. Assim, a via oral tem sido a forma mais segura para a administração de vitamina $\mathrm{K}^{\left({ }^{(94)} \text {. }\right.}$

Para a reversão da anticoagulação pelo uso de warfarina, preconiza-se o seguinte esquema terapêutico $^{(67)}$ :
- $3<$ INR $\leq 5$, sem sangramento significante, sem necessidade de reversão aguda: diminuir a dose ou suspender a warfarina, repetir o INR a cada $48 \mathrm{~h}$, retornar com dose menor, quando o INR atingir a faixa terapêutica;

- $5<$ INR $\leq 9$, sem sangramento significante, sem necessidade de reversão rápida: suspender a warfarina e monitorizar o INR; reiniciar com dose mais baixa, quando o INR atingir a faixa terapêutica, outra opção, principalmente em pacientes com maior risco de sangramento, é suspender a warfarina e administrar Vitamina K (1 - 2,5 mg, via oral), nos pacientes que necessitam de reversão rápida, deve-se administrar vitamina $\mathrm{K}(2-4 \mathrm{mg}$, via oral) e, caso o INR permaneça elevado após 24 $\mathrm{h}$, administrar dose adicional de vitamina K (1 - 2 mg, via oral);

- INR > 9, sem sangramento significante: suspender a warfarina e administrar Vitamina K (3-5 mg, via oral), caso o INR permaneça elevado após 24-48 h, administrar dose adicional de vitamina $\mathrm{K}$, retornar o tratamento com dose reduzida de warfarina, quando o INR estiver novamente na faixa terapêutica; 
- INR > 20 ou em vigência de sangramento importante: suspender a warfarina, administrar Vitamina $\mathrm{K}$ (10 mg, em infusão endovenosa, lenta); dependendo da urgência, pode-se associar plasma fresco ou complexo de protrombina, se necessário, a Vitamina K pode ser repetida a cada $12 \mathrm{~h}$;

- sangramento com risco de vida: suspender a warfarina, administrar Vitamina K $(10 \mathrm{mg}$ em infusão endovenosa lenta), associada à administração de complexo de protrombina, repetir, se necessário, com base no INR.

Atualmente, o complexo de protrombina tem sido preferido ao plasma fresco, pois tem se mostrado mais eficiente na reversão da coagulopatia causada pelo uso de anticoagulante oral, ${ }^{(95,96)}$ além de ser mais seguro quanto ao risco de transmissão de doenças infecciosas.

A duração da anticoagulação deve ser individualizada, caso a caso, levando-se sempre em consideração os riscos e benefícios de tal tratamento. Observar as recomendações abaixo ${ }^{(67)}$ :

- Primeiro episódio de tromboembolismo em um paciente com fator de risco temporário ou reversível (ex: cirurgia, trauma, imobilização, uso de esteróides): anticoagulação por três a seis meses, optando-se geralmente, pela anticoagulação por seis meses.

- Primeiro episódio de tromboembolismo em um paciente sem fator de risco evidente (tromboembolismo idiopático): anticoagulação por, no mínimo, seis meses.

- Primeiro episódio de tromboembolismo em um paciente com fator de risco permanente (ex: neoplasia em atividade, síndrome do anticorpo antifosfolípede, deficiência de antitrombina): anticoagulação por, pelo menos, 12 meses, devendo ser considerada a possibilidade de anticoagulação permanente.

Atualmente, há recomendação para anticoagulação permanente apenas nas situações que seguem ${ }^{(97)}$ :

- Dois ou mais episódios de trombose idiopática.

- Um episódio de trombose idiopática em indivíduos com deficiência de antitrombina ou com síndrome do anticorpo antifosfolípede.

- Pacientes com episódio quase fatal de TEP.

- Pacientes com trombose em veias mesentéricas, cerebrais ou porta.

- Um episódio de trombose em paciente com mais de um defeito genético, que predispõe a fenômenos tromboembólicos.

Ainda não há um consenso sobre a duração da anticoagulação nas seguintes situações, pois um período maior de anticoagulação diminui a recorrência de fenômenos tromboembólicos, mas, também, aumenta o número de complicações, principalmente relacionadas a sangramentos ${ }^{(67,97)}$ :

- primeiro episódio de tromboembolismo em pacientes homozigotos para deficiência do fator $\mathrm{V}$ de Leiden, pacientes com homocisteinemia ou com deficiência de proteína $\mathrm{C}$ ou $\mathrm{S}$;

- tromboembolia recorrente, porém associada a fatores de risco transitórios.

Todas as recomendações estão sujeitas a modificações baseadas nas características individuais de cada paciente, como idade e comorbidades.

\section{7- Trombolíticos}

A indicação absoluta do uso de trombolíticos, no TEP, é a presença de instabilidade hemodinâmica $(9,67)$. No entanto, alguns trabalhos ${ }^{(98,99,100)}$ estendem a indicação dos trombolíticos aos pacientes com pressão arterial normal, porém com disfunção de ventrículo direito pela ecocardiograma.

Os trombolíticos mais utilizados para o tratamento do TEP são a estreptoquinase e o rt-PA, infundidos em veia periférica, nos esquemas posológicos apresentados a seguir.

- Estreptoquinase - 250.000 UI endovenosa durante $30 \mathrm{~min}$ (ataque), seguida de $100.000 \mathrm{UI} / \mathrm{h}$, durante $24 \mathrm{~h}$.

- rt-PA - 100 mg, em infusão venosa contínua, por $2 \mathrm{~h}$.

Após quatro horas do término da administração do trombolítico, deve-se realizar o tempo de tromboplastina parcial ativada (TTPA). Se este estiver menor que duas vezes seu valor basal, deve-se iniciar a heparinização em doses habituais, com o objetivo de manter o TTPA entre 1,5 e 2,5 vezes. A transição para a anticoagulação oral é idêntica à dos pacientes que não receberam trombolíticos.

Entre os benefícios dessa terapêutica, estão a redução do risco de recorrência e a redução da mortalidade pelo TEP ${ }^{(101)}$. Embora o benefício do uso do trombolítico seja proporcional à precocidade com que é introduzido no curso da doença ${ }^{(102)}$, as evidências sustentam seu uso até 14 dias após o início dos sintomas ${ }^{(100)}$.

A principal complicação decorrente do uso de trombolíticos é o sangramento, que ocorre em uma frequiência de até três vezes maior do que no tratamento apenas com heparina. O sangramento mais comum é o associado a procedimentos invasivos, tais como inserções de cateteres, punções venosas e arteriais. O sangramento mais grave e mais temido é o de sistema nervoso central, tendo frequiência de aproximadamente $1 \%{ }^{(79)}$. 
Outras complicações são: febre, reações alérgicas (eritema, urticária e hipotensão), náusea, vômitos, mialgia e dor de cabeça. Estas reações estão mais relacionadas ao uso da estreptoquinase.

Não há contra-indicação absoluta ao uso de trombolíticos diante de um quadro de TEP maciço com instabilidade hemodinâmica. Em todos os casos, deverão, sempre, ser pesados os riscos e benefícios dessa terapia. As contra-indicações relativas estão relacionadas no Quadro $13^{(100)}$.

Assim, devido aos riscos envolvidos com tal tratamento, ele só deverá ser instituído após confirmação diagnóstica, de preferência de forma menos invasiva, para não aumentar a chance de complicação hemorrágica. Entretanto, em pacientes muito instáveis ou quando esses exames não forem disponíveis, dados clínicos e ecocardiográficos compatíveis podem ser suficientes para se instituir o tratamento, visto ser essa uma condição de emergência.

\section{8- Tratamento cirúrgico}

A tromboembolectomia, quando comparada com o tratamento clínico (heparina e trombolíticos),

\section{Quadro 13 - Contra-indicações relativas ao uso dos trombolíticos}

AVC, tumor ou cirurgia intracraniana ou intra-espinhal (nos últimos dois meses)

Doença intracraniana ativa (aneurisma, malformação vascular, neoplasia)

Sangramento interno significativo nos últimos seis meses

Hipertensão arterial sistêmica descontrolada (PAS $\geq 200$ ou $\mathrm{PAD} \geq 110 \mathrm{mmHg}$ )

Diátese hemorrágica, incluindo as associadas com insuficiência renal ou hepática

Cirurgia, biópsia de órgão, parto ou punção não compressível recentes (menos de 10 dias)

Trauma recente (incluindo ressuscitação cardiopulmonar)

Endocardite infecciosa

Gravidez

Retinopatia hemorrágica

Pericardite

Aneurisma ambos na fase aguda do TEP, apresenta como desvantagens sua maior invasividade e sua menor disponibilidade. Entretanto, a tromboembolectomia pode ser utilizada como última alternativa nos casos de TEP maciço com instabilidade hemodinâmica e sem resposta ou com contra-indicação ao uso de trombolítico, desde que haja uma equipe com experiência na técnica e imediatamente disponível ${ }^{(67)}$. Em se tratando de uma cirurgia de urgência, em pacientes em estado extremamente grave, o índice de mortalidade pode chegar até $70 \%{ }^{(103)}$.

Outra opção terapêutica é a colocação de filtros na veia cava inferior, com o objetivo de reduzir a ocorrência de novos fenômenos tromboembólicos. É importante ressaltar que é uma medida profilática, sem participação no trombo pulmonar já instalado. Suas principais indicações seriam as relatadas a seguir ${ }^{(67)}$.

- Contra-indicação ao uso de terapia anticoagulante ou quando já ocorreu alguma complicação da terapia usada.

- Recorrência do tromboembolismo, apesar da terapia anticoagulante adequada,

- Embolia paradoxal.

- Embolia pulmonar crônica, com hipertensão pulmonar.

- Pacientes submetidos à tromboembolectomia ou à tromboendarterectomia.

São consideradas contra-indicações para o filtro de veia cava.

- Presença de anormalidade anatômica do sistema venoso.

- Gravidez.

- Trombo localizado proximalmente ao ponto de implante do filtro.

A tromboendarterectomia tem sido o tratamento preconizado para o TEP crônico, que evoluiu com hipertensão pulmonar e cor pulmonale. Como o trombo crônico fica firmemente aderido à parede interna do vaso, a tentativa de sua retirada por tração promove sua fragmentação e permanência nas regiões distais da artéria pulmonar. Assim, a localização correta do plano anatômico de clivagem é de fundamental importância para a retirada integral do trombo e o posterior sucesso da cirurgia. Um aspecto importante é que a pressão, na artéria pulmonar, mesmo diminuindo, pode não normalizar e, mesmo assim, ainda há melhora clínica ${ }^{(103)}$. Outro ponto fundamental é a prevenção de novas obstruções arteriais, o que pode ocorrer por novos episódios tromboembólicos ou por trombose local, favorecida pela superfície interna do vaso, desprovida de endotélio pós-endarterectomia. Por isso, mais uma vez, torna-se importante o plano de clivagem, 
para reduzir a irregularidade no interior do vaso, e o paciente deve ser novamente heparinizado nas primeiras horas do pós-operatório, tão logo quanto possível. Ainda há discordância, na literatura, quanto à necessidade de implante de filtro de veia cava nos pacientes submetidos à tromboendarterectomia; assim, sua colocação seria mais um cuidado na prevenção de novos episódios tromboembólicos e sem muito risco adicional. A evolução tardia pós-tromboendarterectomia é satisfatória, com manutenção dos resultados a longo prazo ${ }^{(104,105)}$.

\section{7- HIPERTENSÃO ARTERIAL PULMONAR TROMBOEMBÓLICA CRÔNICA}

Geralmente, os dados da literatura sobre seguimento de pacientes com episódio agudo de TEP, restringem-se a valores gasométricos e de tolerância ao exercício. Há pouca caracterização da evolução anatômica e hemodinâmica de tais pacientes ${ }^{(106,107)}$. Assim, pacientes com TEP podem apresentar um espectro de evoluções: resolução anatômica e hemodinâmica completas (minoria dos casos), resolução parcial, associada com perfil clínico normal (maioria dos casos) e progressão para hipertensão pulmonar (em alguns pacientes) ${ }^{(108)}$. Porém, a real incidência da hipertensão arterial pulmonar tromboembólica crônica (HAPTC) não é conhecida, estima-se que seja de aproximadamente $0,1 \%$ dos pacientes que sobrevivem a um episódio de TEP ${ }^{(109)}$.

Já foi demonstrado que, após um episódio agudo de TEP, a pressão da artéria pulmonar leva, aproximadamente, 38 dias para se estabilizar. Em pacientes, nos quais a pressão sistólica da artéria pulmonar, no momento do diagnóstico, é superior a $50 \mathrm{mmHg}$, há um risco até três vezes maior de evolução para HAPTEC ${ }^{(110)}$.

O quadro clínico é caracterizado por um período inicial (meses a anos) assintomático, evoluindo para dispnéia progressiva, hipoxemia e disfunção de ventrículo direito. A velocidade da evolução irá depender de um ou mais dos seguintes fatores: recorrência do tromboembolismo, trombose in situ, alterações na microvasculatura pulmonar e esgotamento dos mecanismos adaptativos do ventrículo direito ${ }^{(108)}$. Como o défice perfusional vai estar sempre presente, a cintilografia de perfusão tem elevada sensibilidade no diagnóstico desses casos ${ }^{(67,111)}$.

A prevenção da HAPTEC pode ser feita com o uso de trombolíticos na fase aguda do TEP, em pacientes selecionados ${ }^{(79)}$. Após o seu desenvolvimento, a tromboendarterectomia é a única opção terapêutica potencialmente eficaz ${ }^{(104,105)}$.

\section{8- TROMBOEMBOLISMO EM GESTANTES}

O diagnóstico de TEP, na gestante, é um grande desafio perante a grande diversidade de sintomas e as dificuldades inerentes à exposição fetal, durante os procedimentos de diagnóstico por imagem. Além disso, o risco de TEV aumenta durante o terceiro trimestre da gestação e nas primeiras semanas após o parto.

No período entre a $2^{\mathrm{a}}$ a $16^{\mathrm{a}}$ semana de gestação, doses de radiação acima de 50 cGy $(1$ cGy $=1$ rad) podem causar retardo no desenvolvimento, malformações e até morte fetal. Já, doses menores que 10 cGy não representam grande risco e nem indicam a interrupção da gravidez ${ }^{(112)}$. Cada procedimento diagnóstico causa uma determinada exposição fetal à radiação ${ }^{(112,113)}$, conforme ilustrado no Quadro 14.

Portanto, na suspeita clínica de TEP, em gestante, deve-se prosseguir a investigação, restringindose, ao mínimo, a exposição fetal à radiação. Assim, a utilização de uma estratégia diagnóstica que inclua radiografia de tórax, duplex ultra-sonografia dos membros inferiores e cintilografia pulmonar, mantém a exposição fetal em níveis de segurança. Quando essa investigação inicial não for suficiente para elucidar o caso, outros exames podem ser realizados, desde que seja analisado seu risco/benefício e que seja assinado um termo de consentimento livre e esclarecido pelos pais ou responsáveis. A tomografia computadorizada helicoidal e a arteriografia pulmonar devem ser realizadas com a utilização de avental de chumbo. No caso da arteriografia, a inserção do cateter deve ser realizada via braquial, em lugar da via femural.

Feito o diagnóstico de TEP, deve-se iniciar heparina (HNF ou HBPM), em doses terapêuticas, durante toda a gestação. A HBPM deverá ser interrompida $12 \mathrm{~h}$ antes do parto ou a partir do início do trabalho

Quadro 14 - Estimativa da radiação absorvida pelo feto nos diferentes procedimentos diagnósticos
Procedimentos

Venografia unilateral sem proteção

Arteriografia pulmonar (via femoral)

Arteriografia pulmonar (via braquial)

Cintilografia de perfusão (Tc-99m)

Cintilografia de ventilação (Tc-99m)

Cintilografia de ventilação (Xe-133)

Radiografia de tórax
Radiação (Gy)

$$
0,314
$$$$
0,405
$$$$
<0,05
$$

$0,006-0,012$

$0,007-0,035$

$0,004-0,019$

$<0,001$ 
de parto. Quando a paciente tiver indicação, previamente ao início de trabalho de parto, de anestesia epidural, a heparina deverá ser interrompida $24 \mathrm{~h}$ antes ${ }^{(114)}$. Caso a paciente seja de alto risco para recidiva de TEV (ex: TVP nas últimas duas semanas), devese substituir a HBPM pela HNF e, esta deverá ser interrompida entre 4 e $6 \mathrm{~h}$ antes do parto ${ }^{(67)}$.

A heparina (padrão e de baixo peso molecular) não ultrapassa a placenta, podendo ser utilizada com segurança ${ }^{(112,113,115)}$. Alguns estudos mostraram comprometimento ósseo pelo uso prolongado (> 1 mês) da heparina, sendo que houve redução subclínica da densidade óssea em 1/3 dos casos e fraturas sintomáticas em menos de $2 \%{ }^{(116)}$. Os cumarínicos não devem ser utilizados durante a gravidez, principalmente entre a $6^{\mathrm{a}}$ e a $12^{\text {a }}$ semana, pelo risco de causarem malformações fetais ${ }^{(117)}$. Dentre as malformações causadas, temos a hipoplasia nasal, a condrodisplasia epifisária e as anomalias no SNC; além disso, hemorragias fetais ou neonatais e óbito intra-uterino podem ocorrer mesmo com níveis adequados de anticoagulação materna.

Após, deve-se iniciar o esquema habitual de anticoagulação com heparina e anticoagulante oral, por, no mínimo, seis semanas. A heparina não é secretada no leite materno e os cumarínicos não produzem efeito anticoagulante, induzido pela amamentação ${ }^{(67,118,119)}$.

Outro aspecto, sempre muito importante, quando se trata de TEV, é a profilaxia, que deve fazer parte integrante da orientação de toda gestante. Naqueles casos, onde há um risco maior para o desenvolvimento de TEV, há várias recomendações individualizadas para cada caso, conforme ilustrado no Quadro $15^{(67)}$

\section{Quadro 15 - Orientações para gestação com risco aumentado de TEV}

Fator de risco

Episódio prévio de TEV em vigência de fator de risco transitório

Episódio prévio de TEV idiopático

Episódio prévio de TEV associado à trombofilia*

Trombofilia sem episódio prévio de TEV

TEV de repetição prévio

Em vigência de anticoagulação, devido a TEV idiopático ou associado à trombofilia

Minidose de HNF

Dose-moderada HNF

Dose terapêutica de HNF

Dose profilática de HBPM

Dose terapêutica de HBPM

\section{Orientação durante a gestação}

Observação clínica rigorosa

Observação clínica rigorosa ou minidose de HNF ou dose moderada de HNF ou dose profilática de HBPM

Observação clínica rigorosa ou ini-dose de HNF ou dose profilática de HBPM

Dose terapêutica de HNF ou dose profilática de HBPM ou dose terapêutica de HBPM

5000 UI, subcutânea, 12-12 h

Suficiente para obter anti Xa*** entre 0,1-0,3 U/ml, subcutânea, $12-12 \mathrm{~h}$

Dose suficiente para manter TTPA em níveis terapêuticos, subcutânea, $12-12 \mathrm{~h}$

5000 U de dalteparina, subcutânea, 24-24 h ou 40U de enoxaparina, subcutânea, 24-24 h ou titular a dose, mantendo anti-Xa entre $0,2-0,6 \mathrm{U} / \mathrm{ml}$

Vide Quadro 11

\footnotetext{
* para pacientes com deficiência de antitrombina, os cuidados devem ser mais intensivos;

** na anticoagulação pós-parto, inicia-se o anticoagulante oral e manter a heparina até que seja atingido um INR > 2,0;

*** para a medida do anti-Xa, o sangue deverá ser colhido 4 h após a administração da heparina
} 


\section{9- CONCLUSÔES}

O TEV apresenta várias dificuldades, não apenas no seu diagnóstico, como, também, no seu tratamento. Além disso, pela possibilidade de deixar seqüelas (HAPTC, alterações neurológicas pela embolia paradoxal, entre outras) e por ser potencialmente fatal até mesmo em pacientes previamente hígidos, convém ressaltar dois pontos primordiais.

O primeiro é a profilaxia, que não será abordada neste capítulo, por ser um assunto extenso, embora não menos importante. As medidas profiláticas para os fenômenos tromboembólicos são realmente efetivas, porém cada paciente deve ser individualizado e estratificado quanto ao risco e, assim, submetido à melhor estratégia profilática.

O segundo é que, para se fazer o diagnóstico de TEV, o primeiro passo é a suspeita clínica. Assim, o médico deverá estar sempre atento para tal possibilidade diagnóstica, para que o TEV possa ser confirmado precocemente, o que aumenta a chance de sobrevida do paciente.

ALVARES F; PÁDUA AI \& TERRA FILHO J. Pulmonary thromboembolism: diagnosis and therapy. Medicina, Ribeirão Preto, 36: 214-240, apr./dec. 2003.

ABSTRACT - Pulmonary embolism is a frequent disease, but it is of low-occurrence diagnosis. This may be justified primarily by the fact that it features low specific symptoms and signs, leading to a delay in clinical suspicion. Furthermore, in spite of the existence of many complementary exams that can help diagnosis, many of them are unavailable in the majority of the medical institutions. This delayed diagnosis has severe repercussions, heading to increasing mortality rates. The main objectives of this revision is to awake about the diagnostic impression, to guide its investigation and its treatment.

UNITERMS - Pulmonary Embolism. Embolism and Thrombosis. Anticoagulation. Diagnosis.

\section{REFERÊNCIAS BIBLIOGRÁFICAS}

1 - GOLDHABER SZ. Medical progress: pulmonary embolism. N Engl J Med 339: 93-104, 1998.

2 - STEIN PD. Pulmonary embolism. Williams \& Wilkins, Baltimore, 1996.

3 - OGER E. Incidence of venous thromboembolism: a community-based study in Western France. Thromb Haemost 83: 657-660, 2000.

4 - HEIT JÁ; MELTON LJ 3rd; LOHSE CM; PETTERSON TM; SILVERSTEIN MD; MOHR DN \& O'FALLON WM. Incidence of venous thromboembolism in hospitalized patients vs community residents. Mayo Clin Proc 76: 1102-1110, 2001.

5 - STEIN PD \& HENRY JW. Prevalence of acute pulmonary embolism among patients in a general hospital and at autopsy. Chest 108: 978-981, 1995.

6 - GOLDHABER SZ; VISANI L \& DE ROSA M. Acute pulmonary embolism: clinical outcomes in the international Cooperative Pulmonary Embolism Registry (ICOPER). Lancet 353: 13861389, 1999.

7 - CARSON JL; KELLEY MA; DUFF A; WEG JG; FULKERSON WJ; PALEVSKY HI; SCHWARTZ JS; THOMPSON BT; POPOVICH J JR \& HOBBINS TE. The clinical course of pulmonary embolism. N Engl J Med 326: 1240-1245, 1992.
8 - BRITISH THORACIC SOCIETY. Suspected acute pulmonary embolism: a practical approach. Thorax 52: 1S -24S, 1997.

9 - BRITISH THORACIC SOCIETY. Guidelines for the management of suspected acute pulmonary embolism. Thorax 58: 470-484, 2003.

10 - ROSENDAAL FR. Venous thrombosis: a multicausal disease. Lancet 353: 1167-1173, 1999.

11 - HEIT JÁ; SILVERSTEIN MD; MOHR DN; PETTERSON TM; O'FALLON WM \& MELTON LJ 3rd. Risk factors for deep vein thrombosis and pulmonary embolism: a population-based casecontrol study. Arch Intern Med 160: 809-815, 2000.

12 - HEIT JÁ; SILVERSTEIN MD \& MOHR DN. The epidemiology of venous thromboembolism in the community. Thromb Haemost 86: 452-463, 2001.

13 - TSAI AW; CUSHMAN M; ROSAMOND WD; HECKBERT SR; POLAK JF \& FOLSOM AR. Cardiovascular risk factors and venous thromboembolism etiology. Arch Intern Med 162: 1182-1189, 2002.

14 - MORRELL MT. The relationship between leg-vein thrombosis and pulmonary emboli at autopsy. Br J Surg 60: 306, 1973.

15 - DORFMAN GS; CRONAN JJ; TUPPER TB; MESSERSMITH RN; DENNY DF \& LEE CH. Occult pulmonary embolism: a common occurrence in deep venous thrombosis. AJR Am J Roentgenol 148: 263-266, 1987. 
16 - MISMETTI P; JUILLARD-DELSART D; TARDY B; LAPORTESIMITSIDIS S \& DECOUSUS $H$. Evaluation of the risk of venous thromboembolism in the medical patients. Therapie 53: 565570,1998

17 - SELIGSOHN U \& LUBETSKY A. Genetic susceptibility to venous thrombosis. N Engl J Med 344: 1222-1231, 2001.

18 - GREAVES M \& BAGLIN T. Laboratory testing for heritable thrombophilia: impact on clinical management of thrombotic disease. Br J Haematol 109: 699-703, 2000.

19 - BRITISH COMMITTEE FOR STANDARDS IN HAEMATOLOGY. Investigation and management of heritable thrombophilia. $\mathbf{B r}$ J Haematol 114: 512-528, 2001.

20 - GRAU E; REAL E; MEDRANO J; PASTOR E \& SELFA S Recurrent venous thromboembolism in a Spanish population: incidence, risk factors, and management in a hospital setting. Thromb Res 96: 335-341,1999.

21 - PRANDONI P; LENSING AW; BULLER HR; COGO A; PRINS $\mathrm{MH}$; CATTELAN AM; CUPPINI S; NOVENTA F \& ten CATE JW. Deep-vein thrombosis and the incidence of subsequent symptomatic cancer. N Engl J Med 327: 1128-1133, 1992.

22 - SORENSEN HT; MELLEMKJAER L; STEFFENSEN FH; OLSEN $\mathrm{JH} \&$ NIELSEN GL. The risk of a diagnosis of cancer after primary deep venous thrombosis or pulmonary embolism. $\mathbf{N}$ Engl J Med 338: 1169-1173,1998.

23 - HETTIARACHCHI RJ; LOK J; PRINS MH; BULLER HR \& PRANDONI P. Undiagnosed malignancy in patients with venous thromboembolism. Cancer 83: 180-185, 1998.

24 - STEIN PD; WILLIS PW 3rd \& DEMETS DL. History and physical examination in acute pulmonary embolism in patients without preexisting cardiac or pulmonary disease. Am J Cardiol 47: 218-223, 1981.

25 - The PIOPED Investigators. Value of the ventilation/perfusion scan in acute pulmonary embolism: Results of the Prospective Investigation of Pulmonary Embolism Diagnosis (PIOPED). JAMA 263: 2753-2759, 1990.

26 - STEIN PD; TERRIN ML; HALES CA; PALEVSKY HI; SALTZMAN HÁ; THOMPSON BT \& WEG JG. Clinical, laboratory, roentgenographic and electrocardiographic findings in patients with acute pulmonary embolism and no pre-existing cardiac or pulmonary disease. Chest 100: 598-603, 1991.

27 - HYER TM. Venous Thromboembolism. Am J Respir Crit Care Med 159: 1-14, 1999.

28 - EGERMAYER P \& PEACOCK AJ. Is pulmonary embolism a common cause of chronic pulmonary hypertension? Limitations of the embolic hypothesis. Eur Respir J 15: 440-448, 2000.

29 - AFONSO JE. Alterações circulatórias do pulmão. In: TARANTINO AB. Doenças pulmonares, $4^{\mathrm{a}}$. edição, Guanabara Koogan, Rio de Janeiro, p. 877-895, 1997.

30 - WELLS PS; ANDERSON DR; RODGER M; GINSBERG JS; KEARON C; GENT M; TURPIE AG; BORMANIS J; WEITZ J; CHAMBERLAIN M; BOWIE D; BARNES D \& HIRSH J. Derivation of a simple clinical model to categorize patients probability of pulmonary embolism: increasing the models utility with the SimpliRED D-dimer. Thromb Haemost 83: 416420,2000 .
31 - FEDULLO PF \& TAPSON VF. Evaluation of suspected pulmonary embolism. N Engl J Med 349: 1247-1256, 2003.

32 - WORSLEY DF; ALAVI A; ARONCHICK JM; CHEN JT; GREENSPAN RH \& RAVIN CE. Chest radiographic findings in patients with acute pulmonary embolism: observations from the PIOPED Study. Radiology 189: 133-136, 1993.

33 - FREYBURGER G; TRILLAUD H; LABROUCHE S; GAUTHIER $P$; JAVORSCHI S; BERNARD P \& GRENIER N. D-dimer strategy in thrombosis exclusion - a gold standard study in 100 patients suspected of deep venous thrombosis or pulmonary embolism: 8 DD methods compared. Thromb Haemost 79: 32-37, 1998.

34 - QUINN DA; FOGEL RB; SMITH CD; LAPOSATA M; TAYLOR THOMPSON B; JOHNSON SM; WALTMAN AC \& HALES CA. D-Dimers in the diagnosis of pulmonary embolism. Am J Respir Crit Care Med 159: 1445-1449, 1999.

35 - CONSENSUS COMMITTEE ON PULMONARY EMBOLISM. AMERICAN COLLEGE OF CHEST PHYSICIANS (ACCP). Opinions regarding the diagnosis and management of venous thromboembolic disease. Chest 113: 499-504, 1998.

36 - STEIN PD; DALEN JE; McINTYRE KM; SASAHARA AA; WENGER NK \& WILLIS PW 3rd. The electocardiogram in acute pulmonary embolism. Prog Cardiovasc Dis 17: 247-257, 1975.

37 -RIBEIRO A; LINDMARKERP; JUHLIN-DANNFELTA; JOHNSSON $\mathrm{H}$ \& JORFELDT L. Echocardiography Doppler in pulmonary embolism: right ventricular dysfunction as a predictor of mortality rate. Am Heart J 134: 479-487, 1997.

38 - GRIFONI S; OLIVOTTO I; CECCHINI P; PIERALLI F; CAMAITIA; SANTORO G; CONTI A; AGNELLI G \& BERNI G. Short-term clinical outcome of patients with acute pulmonary embolism, normal blood pressure, and echocardiographic right ventricular dysfunction. Circulation 101: 2817-2822, 2000.

39 - VIEILLARD-BARON A; PAGE B; AUGARDE R; PRIN S; QANADLI S; BEAUCHET A; DUBOURG O \& JARDIN F. Acute cor pulmonale in massive pulmonary embolism: incidence, echocardiographic pattern, clinical implications and recovery rate. Intensive Care Med 27: 1481-1486, 2001.

40 - GOLDHABER SZ; VISANI L \& DE ROSA M. Acute pulmonary embolism: clinical outcomes in the International Cooperative Pulmonary Embolism Registry (ICOPER). Lancet 353: 1386$1389,1999$.

41 - RIBEIRO A; LINDMARKER P; JOHNSSON H; JUHLIN-DANNFELT A \& JORFELDT L. Pulmonary embolism: one-year follow-up with echocardiography doppler and five-year survival analysis. Circulation 99: 1325-1330, 1999.

42 - GOLDHABER SZ. Echocardiography in the management of pulmonary embolism. Ann Intern Med 136: 691-700, 2002.

43 - CHARTIER L; BÉRA J; DELOMEZ M; ASSEMAN P; BEREGI JP; BAUCHART JJ; WAREMBOURG H \& THERY C. Free-floating thrombi in the right heart: diagnosis, management, and prognostic indexes in 38 consecutive patients. Circulation 99: 2779-2783, 1999.

44 - MEYER T; BINDER L; HRUSKA N; LUTHE H \& BUCHWALD AB. Cardiac troponin I elevation in acute pulmonary embolism is associated with right ventricular dysfunction. J Am Coll Cardiol 36: 1632-1636, 2000. 
45 - GIANNITSIS E; MÜLLER-BARDORFF M; KUROWSKI V; WEIDTMANN B; WIEGAND U; KAMPMANN M \& KATUS HA. Independent prognostic value of cardiac troponin $T$ in patients with confirmed pulmonary embolism. Circulation 102: 211-217, 2000.

46 - WOLFE MW; LEE RT; FELDSTEIN ML; PARKER JA; COME PC \& GOLDHABER SZ. Prognostic significance of right ventricular hypokinesis and perfusion lung scan defects in pulmonary embolism. Am Heart J 127: 1371-1375, 1994.

47 - RIBEIRO A; JUHLIN-DANNFELT A; BRODIN LA; HOLMGREN A \& JORFELDT L. Pulmonary embolism: relation between the degree of right ventricle overload and the extent of perfusion defects. Am Heart J 135: 868-874, 1998.

48 - RATHBUN SW; RASKOB GE \& WHITSETT TL. Sensitivity and specificity of helicoidal computed tomography in the diagnosis of pulmonary embolism: a systematic review. Ann Intern Med 132: 227-232, 2000.

49 - HIORNSN MP \& MAYO JR. Spiral computed tomography for acute pulmonary embolism. Can Assoc Radiol J 53: 258268, 2002.

50 - RUIZ Y; CABALLERO P; CANIEGO JL; FRIERA A; OLIVERA MJ; TAGARRO D \& ALVAREZ-SALA R. Prospective comparison of helical CT with angiography in pulmonary embolism: global and selective vascular territory analysis. Interobserver agreement. Eur Radiol 13: 823-829, 2003.

51 - OSER RF; ZUKERMAN DA; GUTIERREZ FR \& BRINK JA. Anatomic distribution of pulmonary emboli at pulmonary angiography: implications for cross-sectional imaging. Radiology 199: 31-35, 1996.

52 - STEIN PD \& HENRY JW. Prevalence of acute pulmonary embolism in central and subsegmental pulmonary arteries and relation to probability interpretation of ventilation/perfusion lung scans. Chest 111: 1246-1248, 1997.

53 - PATEL S; KAZEROONI EA \& CASCADE PN. Pulmonary embolism: optimization of small pulmonary artery visualization at multi-detector row CT. Radiology 227: 455-460, 2003.

54 - MUSSET D; PARENT F; MEYER G; MAITRE S; GIRARD P; LEROYER C; REVEL MP; CARETTE MF; LAURENT M; CHARBONNIER B; LAURENT F; MAL H; NONENT M; LANCAR R; GRENIER P \& SIMONNEAU G; EVALUATION DU SCANNER SPIRALE DANS L'EMBOLIE PULMONAIRE STUDY GROUP. Diagnostic strategy for patients with suspected pulmonary embolism: a prospective multicentre outcome study. Lancet 360: 1914-1920, 2002.

55 - PERRIER A; HOWARTH N; DIDIER D; LOUBEYRE P; UNGER PF; DE MOERLOOSE P; SLOSMAN D; JUNOD A \& BOUNAMEAUX H. Performance of helical computed tomography in unselected outpatients with suspected pulmonary embolism. Ann Intern Med 135: 88-97, 2001.

56 - STEIN PD; ATHANASOULIS C; ALAVI A; GREENSPAN RH; HALES CA; SALTZMAN HA; VREIM CE; TERRIN ML \& WEG JG. Complications and validity of pulmonary angiography in acute pulmonary embolism. Circulation 85: 462-468, 1992.

57 - NILSSON T; CARLSSON A \& MARE K. Pulmonary angiography: a safe procedure with modern contrast media and technique. Eur Radiol 8: 86-89, 1998.

58 - KEARON C; GINSBERG JS \& HIRSH J. The role of venous ultrasonography in the diagnosis of suspected deep venous thrombosis and pulmonary embolism. Ann Intern Med 129: 1044-1049, 1998.
59 - TURKSTRA F; KUJJER PJM; van BEEK EJ; BRANDJES DP; ten CATE JW \& BULLER HR. Diagnostic utility of ultrasonography of leg veins in patients suspected of having pulmonary embolism. Ann Intern Med 126: 775-781, 1997.

60 - GIRARD P; MUSSET D; PARENT F; MAITRE S; PHLIPPOTEAU $C$ \& SIMONNEAU G. High prevalence of detectable deep venous thrombosis in patients with acute pulmonary embolism. Chest 116: 903-908, 1999.

61 - KATZ DS; LOUD PA; BRUCE D; GITTLEMAN AM; MUELLER R; KLIPPENSTEIN DL \& GROSSMAN ZD. Combined CT venography and pulmonary angiography: a comprehensive review. Radiographics 22: S3-19, 2002.

62 - LIPPMANN ML \& FEIN AM. The diagnosis of acute pulmonary embolism in patients with COPD. Chest 104: 983-984, 1993.

63 - GOODMAN LR; LIPCHIK RJ; KUZO RS; LIUY; MCAULIFFE TL \& O'BRIEN DJ. Subsequent pulmonary embolism: risk after a negative helicoidal CT pulmonary angiogram - prospective comparison with scintigraphy. Radiology 215: 535-542, 2000.

64 - AUGER WR; FEDULLO PF; MOSER KM; BUCHBINDER M \& PETERSON KL. Chronic major-vessel thromboembolic pulmonary artery obstruction: appearance at angiography. Radiology 182: 393-398, 1992.

65 - ASIF A; PRESTON RA \& ROTH D. Radiocontrast-induced nephropathy. Am J Ther 10: 137-147, 2003.

66 - RASCHKE RA; REILLY BM; GUIDRY JR; FONTANA JR \& SRINIVAS $S$. The weight-based heparin dosing nomogram compared with a "standard care" nomogram. A randomized controlled trial. Ann Intern Med 119: 874-881, 1993.

67 -SIXTH ACCP CONSENSUS CONFERENCE ON ANTITHROMBOTIC THERAPY. Chest 119: 1S-370S, 2001.

68 - LEVINE MN; RASKOB G; LANDEFELD S \& KEARON C. Hemorrhagic complications of anticoagulant treatment. Chest 114: 511S-523S, 1998.

69 - CINES DB; TOMASKI A \& TANNENBAUM S. Immune endothelial-cell injury in heparin-associated thrombocytopenia. $\mathbf{N}$ Engl J Med 316: 581-589, 1987.

70 - DUKES GE JR; SANDERS SW; RUSSO J JR; SWENSON E; BURNAKIS TG; SAFFLE JR \& WARDEN GD. Transaminase elevations in patients receiving bovine or porcine heparin. Ann Intern Med 100: 646-650, 1984.

71 - EDES TE \& SUNDERRAJAN EV. Heparin-induced hipercalemia. Arch Intern Med 145: 1070-1072, 1985.

72 - WHITE PW; SADD JR \& NENSEL RE. Thrombotic complications of heparin therapy. Ann Surg 190: 595-608, 1979.

73 - PROTAMINE SULFATE ANTIHEPARIN AGENTS 20:12.08. In: MCEVOY G; LITVAK K \& WELSH OH, eds. AHFS Drug Information 1999. American Society of Heath-System Pharmacists, Bethesda, MD, p. 1265-1267, 1999.

74 - HULL RD; RASKOB GE; BRANDT RF; PINEO GF; STEIN PD \& MAH AF. Low-molecular-weight heparin vs heparin in the treatment of patients with pulmonary embolism. Arch Intern Med 160: 229-236, 2000.

75 - CASELE HL; LAIFER SA; WOELKERS DA \& VENKATARAMANAN R. Changes in the pharmacokinetics of the low-molecular-weight heparin enoxaparin sodium during pregnancy. Am J Obstet Gynecol 181: 1113-1117, 1999. 
76 - LAPOSATA M; GREEN D; VAN COTT EM; BARROWCLIFFE TW; GOODNIGTH SH \& SOSOLIK RC. College of American Pathologists Conference XXXI on laboratory monitoring of anticoagulant therapy: the clinical use and laboratory monitoring of low-molecular-weight heparin, danaparoid, hirudin and related compounds, and argatroban. Arch Pathol Lab Med 122: 799-807,1998.

77 - BONEU B \& DE MOERLOOSE P. How and when to monitor a patient treated with low molecular weight heparin. Semin Thromb Hemost 27: 519-522, 2001.

78 - WAKEFIELD TW; ANDREWS PC; WROBLESKI SK; KADELL AM; TEJWANI S; HULIN MS \& STANLEY JC. A [+18RGD] protamine variant for nontoxic and effective reversal of conventional heparin and low-molecular-weight heparin anticoagulation. J Surg Res 63: 280-286, 1996.

79 - DIETRICH CP; SHINJO SK; MORAES FA; CASTRO RA; MENDES A; GOUVEA TC \& NADER HB. Structural features and bleeding activity of commercial low molecular weight heparins: neutralization by ATP and protamine. Semin Thromb Hemost 25: 43-50, 1999.

80 - PRANDONI P; LENSING AW; BULLER HR; CARTAM; COGO A; VIGO M; CASARA D; RUOLA \& TEN CATE JW. Comparison of subcutaneous low-molecular-weight heparin with intravenous standard heparin in proximal deep-vein thrombosis. Lancet 339: 441-445, 1992.

81 - GOULD MK; DEMBITZERAD; DOYLE RL; HASTIE TJ \& GARBER $A M$. Low-molecular-weight heparins compared with unfractionated heparin for treatment of acute deep venous thrombosis. A meta-analysis of randomized, controlled trials. Ann Intern Med 130: 800-809,1999.

82 - SCIUMBATA T; CARETTO P; PIROVANO P; POZZI P; CREMONESI P; GALIMBERTI G; LEONI F \& MARCUCCI F. Treatment with modified heparins inhibits experimental metastasis formation and leads, in some animals, to long-term survival. Invasion Metastasis 16: 132-143, 1996.

83 - NORRBY K. Heparin and angiogenesis: a low-molecularweight fraction inhibits and a high-molecular-weight fraction stimulates angiogenesis systemically. Haemostasis 23: $141 \mathrm{~S}-149 \mathrm{~S}, 1993$.

84 - SCHIELE F; VUILLEMENOT A; KRAMARZ P; KIEFFER Y; ANGUENOT T; BERNARD Y \& BASSAND JP. Use of recombinant hirudin as antithrombotic treatment in patients with heparin-induced thrombocytopenia. Am J Hematol 50: 2025, 1995

85 - O'REILLY RA \& AGGELER PM. Studies on coumarin anticoagulant drugs: initiation of Warfarin therapy with a loading dose. Circulation 38: 169-177, 1968.

86 - HARRISON L; JOHNSTON M; MASSICOTTE MP; CROWTHER $\mathrm{M}$; MOFFAT K \& HIRSH J. Comparison of 5-mg and 10-mg loading doses in initiation of warfarin therapy. Ann Intern Med 126: 133-136, 1997.

87 - CROWTHER MA; GINSBERG JS; JULIAN J; DENBURG J; HIRSH J; DOUKETIS J; LASKIN C; FORTIN P; ANDERSON D; KEARON C; CLARKEA; GEERTS W; FORGIE M; GREEN D; COSTANTINI L; YACURA W; WILSON S; GENT M; KOVACS MJ. A comparison of two intensities of warfarin for the prevention of recurrent thombosis in patients with the antiphospholipid antibody syndrome. N Engl J Med 349: 1133-1138, 2003.
88 - DALE J; MYHRE E \& LOEW D. Bleeding during acetylsalicylic acid and anticoagulant therapy in patients with reduced platelet reactivity after aortic valve replacement. Am Heart J 99: 746-751, 1980.

89 - SCHULMAN S \& HENRIKSSON K. Interaction of ibuprofen and Warfarin on primary hemostasis. $\mathbf{B r} \mathbf{J}$ Rheumatol 38: 46-49, 1989.

90 - FIHN SD; MCDONELL M; MARTIN D; HENIKOFF J; VERMES D; KENT D \& WHITE RH. Risk factors for complications of chronic anticoagulation. A multicenter study. Warfarin Optimized Outpatient Follow-up Study Group. Ann Intern Med 118: 511-520, 1993.

91 - LAUNBJERG J; EGEBLAD H; HEAFJ; NIELSEN NH; FUGLEHOLM AM \& LADEFOGED K. Bleeding complications to oral anticoagulant therapy: multivariate analysis of 1010 treatment years in 551 outpatients. J Intern Med 229: 351-355, 1991.

92 - BROEKMANS AW; BERTINARM; LOELIGER EA; HOFMANN V \& KLINGEMANN HG. Protein C and the development of skin necrosis during anticoagulant therapy. Thromb Haemost 49: 251, 1983.

93 - GRIMAUDO V; GUEISSAZ F; HAUERT J; SARRAJ A; KRUITHOF EK \& BACHMANN F. Necrosis of skin induced by coumarin in a patient deficient in protein S. BMJ 298: 233-234, 1989.

94 - CROWTHER MA; DONOVAN D; HARRISON L; MCGINNIS J \& GINSBERG J. Low-dose oral vitamin K reliably reverses overanticoagulation due to warfarin. Thromb Haemost 79: 1116-1118, 1998.

95 - MAKRIS M; GREAVES M; PHILLIPS WS; KITCHEN S; ROSENDAAL FR \& PRESTON EF. Emergency oral anticoagulant reversal: the relative efficacy of infusions of fresh frozen plasma and clotting factor concentrate on correction of the coagulopathy. Thromb Haemost 77: 477-480, 1997.

96 - HELLSTERN P; MUNTEAN W; SCHRAMM W; SEIFRIED E \& SOLHEIM BG. Practical guidelines for the clinical use of plasma. Thromb Res 107: 53S -57S, 2002.

97 - BAUER KA. Management of thrombophilia. J Thromb Haemost 1: 1429-1434, 2003.

98 - GOLDHABER SZ. Thrombolytic therapy in venous thromboembolism: clinical trials and current indications. Clin Chest Med 16: 307-320, 1995.

99 - CANNON CP \& GOLDHABER SZ. Cardiovascular risk stratification of pulmonary embolism. Am J Cardiol 78: 11491151, 1996.

100 - ARCASOY SM \& KREIT JW. Thrombolytic therapy of pulmonary embolism. A comprehensive review of current evidence. Chest 115: 1695-1707, 1999.

101 - KONSTANTINIDES S; GEIBELA; OLSCHEWSKI M; HEINRICH F; GROSSER K; RAUBER K; IVERSEN S; REDECKER M; KIENAST J; JUST H \& KASPER W. Association between thrombolytic treatment and the prognosis of hemodynamically stable patients with major pulmonary embolism: results of a multicenter registry. Circulation 96: 882-888, 1997.

102 - DANIELS LB; PARKER JA; PATEL SR; GRODSTEIN F \& GOLDHABER SZ. Relation of duration of symptoms with response to thrombolytic therapy in pulmonary embolism. Am J Cardiol 80: 184-188, 1997. 
103 - CLARKE DB \& ABRAMS LD. Pulmonary embolectomy: a 25 year experience. J Thorac Cardiovasc Surg 92: 442445, 1986.

104 - JATENE FB; PÊGO-FERNANDES PM; POVEDA S; MONTEIRO R; CUKIER A; MADY C \& JATENE AD. Tratamento cirúrgico da embolia pulmonar crônica: análise da experiência inicial. Rev Bras Cir Cardiovasc 10: 70-76, 1995.

105 - ARCHIBALD CJ; AUGER WR; FEDULLO PF; CHANNICK RN; KERR KM; JAMIESON SW; KAPELANSKI DP; WATT CN \& MOSER KM. Long-term outcome after pulmonary thromboendarterectomy. Am J Respir Crit Care Med 160: 523-528, 1999.

106 - MOSER KM. Venous Thromboembolism. Am Rev Respir Dis 141: 235-249, 1990.

107 - PETERSON KL. Acute pulmonary embolism. Has its evolution been redefined. Circulation 99: 1280-1283, 1999.

108 - FEDULLO PF; RUBIN LJ; KERR KM;AUGER WR \& CHANNICK RN. The natural history of acute and chronic thromboembolic disease: the search for the missing link. Eur Respir J 15: 435-437, 2000.

109 - FEDULLO PF; AUGER WR; CHANNICK RN; MOSER KM \& JAMIESON SW. Chronic thromboembolic pulmonary hypertension. Clin Chest Med 16: 353-374, 1995.

110 - RIBEIRO A; LINDMARKER P; JOHNSSON H; JUHLINDANNFELT A \& JORFELDT L. Pulmonary embolism. Oneyear follow-up with echocardiography doppler and fiveyear survival analysis. Circulation 99: 1325-1330, 1999.

111 - RYAN KL; FEDULLO PF; DAVIS GB; VASQUEZ TE \& MOSER $K M$. Perfusion scan findings understate the severity of angiographic and hemodynamic compromise in chronic thromboembolic pulmonary hipertension. Chest 93: 1180$1185,1988$.
112 - FLESSA HC; KAPSTROM AB; GLUECK HI \& WILL JJ. Placental transport of heparin. Am J Obstet Gynecol 93: 570573, 1965.

113 - GINSBERG JS; HIRSH J; TURNER DC; LEVINE MN \& BURROWS R. Risks to the fetus of anticoagulant therapy during pregnancy. Thromb Haemost 61: 197-203, 1989.

114 - CONARD J; HORELLOU MH \& SAMAMA MM. Inherited thrombophilia and gestational venous thromboembolism. Semin Thromb Hemost 29: 131-142, 2003.

115 - SANSON BJ; LENSING AW; PRINS MH; GINSBERG JS; BARKAGAN ZS; LAVENNE-PARDONGE E; BRENNER B; DULITZKY M; NIELSEN JD; BODA Z; TURI S; MAC GILLAVRY MR; HAMULYAK K; THEUNISSEN IM; HUNT BJ \& BULLER HR. Safety of low-molecular-weight heparin in pregnancy: a systematic review. Thromb Haemost 81: 668-672, 1999.

116 - NELSON-PIERCY C; LETSKY EA \& DE SWIET M. Low-molecular-weight heparin for obstetric thromboprophylaxis: experience of sixty-nine pregnancies in sixty-one women at high risk. Am J Obstet Gynecol 176: 1062-1068, 1997.

117 - DOUKETIS JD; GINSBERG JS; BURROWS RF; DUKU EK; WEBBER CE \& BRILL-EDWARDS P. The effects of longterm heparin therapy during pregnancy on bone density. A prospective matched cohort study. Thromb Haemost 75: 254-257, 1996.

118 - HALL JG; PAULI RM \& WILSON KM. Maternal and fetal sequelae of anticoagulation during pregnancy. Am J Med 68: 122-140, 1980.

119 - MCKENNA R; COLE ER \& VASAN U. Is warfarin sodium contraindicated in the lactating mother? J Pediatr 103: 325-327, 1983. 